\title{
Neurotransmitter organization of the nucleus of Edinger-Westphal and its projection to the avian ciliary ganglion
}

\author{
ANTON REINER, ${ }^{1}$ JONATHAN T. ERICHSEN,${ }^{2}$ JOHN B. CABOT, ${ }^{2}$ CRAIG EVINGER, ${ }^{2}$ \\ MALINDA E.C. FITZGERALD, ${ }^{1}$ AND HARVEY J. KARTEN ${ }^{3}$ \\ ${ }^{1}$ Department of Anatomy and Neurobiology, University of Tennessee, Memphis \\ ${ }^{2}$ Department of Neurobiology and Behavior, State University of New York at Stony Brook, Stony Brook \\ ${ }^{3}$ Department of Neurosciences, University of California at San Diego, La Jolla
}

(ReCEived October 22, 1990; ACCEPTEd December 21, 1990)

\begin{abstract}
Two morphologically distinct types of preganglionic endings are observed in the avian ciliary ganglion: boutonal and cap-like. Boutonal endings synapse on ciliary ganglion neurons (called choroidal neurons) innervating choroidal blood vessels, while cap-like endings synapse on ciliary ganglion neurons (called ciliary neurons) controlling the lens and pupil. Some of both types of preganglionic endings contain the neuropeptides substance P (SP) and/or leucine-enkephalin (LENK). Although both types of preganglionic terminals are also known to be cholinergic, there has been no direct evidence that SP and LENK are found in cholinergic endings in the ciliary ganglion. The present studies in pigeons, which involved the use of singleand double-label immunohistochemical techniques, were undertaken to examine this issue, as well as to (1) determine the relative percentages of the boutonal and cap-like endings that contain SP, LENK, or both SP and LENK; and (2) determine if the two different types of terminals in the ciliary ganglion arise from different subdivisions of the nucleus of Edinger-Westphal (EW).

Single- and double-label immunohistochemical studies revealed that all neurons of EW, regardless of whether they contained immunohistochemically detectible amounts of SP or LENK, are cholinergic. In the medial subdivision of EW (EWM), which was found to contain approximately 700 neurons, $20.2 \%$ of these neurons were observed to contain both SP and LENK, while $11.6 \%$ were observed to contain SP only and $10.7 \%$ were observed to contain LENK only. In contrast, in lateral EW (EWL), which was found to contain approximately 500 neurons, $16.2 \%$ of the neurons were observed to contain both SP and LENK, while $19.2 \%$ of the neurons were observed to contain SP only and $12.6 \%$ were observed to contain LENK only. Retrograde-labeling studies involving horseradish peroxidase injections into the ciliary ganglion revealed that EW was the sole source of input to the ciliary ganglion and all, or nearly all, neurons in EW innervate the ciliary ganglion.

Immunohistochemical labeling of the ciliary ganglion neurons with an antiserum against choline acetyltransferase revealed that approximately 900 choroidal neurons and approximately 600 ciliary neurons are present in the ganglion, all of which receive cholinergic preganglionic endings. Of the choroidal neurons, $94 \%$ receive butonal terminals containing both SP and LENK, while only $2 \%$ receive SP+ only boutonal endings and $2 \%$ receive LENK + only butonal endings. Of the ciliary neurons, $25 \%$ receive cap-like endings containing both SP and LENK, $30 \%$ receive cap-like endings containing only SP and $3 \%$ receive cap-like endings containing only LENK. Total unilateral lesions of EW resulted in the loss of all SP+ or LENK+ terminals in the ipsilateral ganglion. Subtotal EW lesions that spared either part of EWM or part of EWL revealed that boutonal endings arise from EWM neurons and cap-like endings from EWL neurons.

The present results suggest that the choroidal neurons, which regulate choroidal blood flow, may be relatively uniform in their functional properties since they nearly all receive boutonal endings from EWM that co-contain SP, LENK, and acetylcholine. In contrast, the ciliary neurons, which receive their preganglionic input from EWL, may consist of at least three major functionally distinct subgroups: (1) those receiving SP/LENK/acetylcholine-containing cap-like endings; (2) those receiving SP/acetylcholine-containing cap-like endings; and (3) those receiving acetylcholine-containing cap-like endings. The functional diversity of ciliary neurons may in part be related to the fact that some ciliary neurons innervate the iris and others the ciliary body.
\end{abstract}

Keywords: Substance P, Enkephalin, Acetylcholine, Co-occurrence, Accommodation, Pupil, Choroid, Immunohistochemistry

Reprint requests to: Anton Reiner, Department of Anatomy and Neurobiology, University of Tennessee, 875 Monroe Avenue, Memphis, TN 38163, USA. 


\section{Introduction}

The avian ciliary ganglion consists of two morphologically distinct neuronal populations (Fig. 1): ciliary neurons that innervate the constrictor muscle of the iris or the ciliary body (thereby controlling pupilloconstriction or accommodation, respectively), and choroidal neurons that innervate the choroidal vasculature (thereby controlling choroidal blood flow) (Hess, 1965; Pilar \& Vaughn, 1969; Landmesser \& Pilar, 1970, 1978; Marwitt et al., 1971; Pilar et al., 1980; Fitzgerald et al., 1990b). These two types of neurons also differ in terms of the morphology of the preganglionic terminals that synapse upon them. Each ciliary neuron receives a single cap-like ending that encapsulates the pole of the neuron from which the axon hillock arises (Hess, 1965; Landmesser \& Pilar, 1970, 1978; Marwitt et al., 1971). In contrast, each choroidal neuron receives many boutonal endings, which cover much of the soma of these neurons (Hess, 1965; Landmesser \& Pilar, 1970, 1978; Marwitt et al., 1971). Both types of endings arise from the visceral subdivision of the oculomotor nuclear complex, termed the nucleus of Edinger-Westphal (EW) (Karten \& Hodos, 1967; Cowan \& Wenger, 1968; Narayanan \& Narayanan, 1976; Lyman \& Mugnaini, 1980). Each choroidal neuron appears to receive inputs from several neurons of EW, while each ciliary neuron received input from only a single EW neuron, although several ciliary neurons may receive input from the same EW neuron (Landmesser \& Pilar, 1970, 1978; Dryer \& Chiappinelli, 1985b). Pilar and his co-workers have shown that transmission between preganglionic fibers and ciliary ganglion neurons is cholinergic (Hess, 1965; Landmesser \& Pilar, 1970, 1978; Pilar \& Tuttle, 1982). Based on immunohistochemical studies, Erichsen et al. $(1982 a, b)$ reported that the neuropeptide substance $\mathrm{P}$ (SP) and the enkephalin (ENK) pentapeptides, leucine-enkephalin (LENK) and methionine-enkephalin (MENK), are also present in both types of preganglionic endings in the ciliary ganglion. Erichsen et al. (1982b) further demonstrated that SP and ENK themselves co-occur in many preganglionic endings of both types. These results suggested that at least some of the cholinergic endings in the ciliary ganglion also contain one or more neuropeptides. Biochemical studies have confirmed that the SPimmunoreactive substance and the LENK- and MENK-immunoreactive substances found in the avian ciliary ganglion are indistinguishable by high-performance liquid chromatography from mammalian SP, LENK and MENK, respectively (White et al., 1985).

Several lines of evidence indicate a functional role for SP and ENK in neurotransmission in the ciliary ganglion. For example, ultrastructural studies indicate that the SP and ENK are localized to large dense-core vesicles in preganglionic terminals of the ciliary ganglion, thus suggesting that preganglionic endings are capable of releasing both SP and ENK (Erichsen et al., 1982a). Further neurophysiological and receptor-binding studies directly support the idea that SP and ENK influence the activity of ciliary ganglion neurons (Role, 1984; Dryer \& Chiappinelli, $1985 a, b$; Margiotta \& Berg, 1986). Given the presumptive cooccurrence of SP and ENK in cholinergic preganglionic endings of the ciliary ganglion, it is also interesting that two neurophysiological studies have provided evidence for an influence of SP on cholinergic transmission in the ciliary ganglion (Role, 1984; Margiotta \& Berg, 1986).

Further clarification of the role of SP and ENK in the cili- ary ganglion requires more detailed anatomical information on the extent to which these neuropeptides co-occur with each other and with acetylcholine in the preganglionic terminals ending on the different types of neurons in the ciliary ganglion. For example, it is important to know the pattern of co-localization of SP and ENK with acetylcholine in the boutonal endings on choroidal neurons as compared to the cap-like endings on ciliary neurons. Similarly, it is important to know the pattern of such co-localization for ciliary neurons innervating the iris as compared to ciliary neurons innervating the muscles of accommodation. Such information will help in the interpretation of the electrophysiological data concerning the effects of SP and ENK on neurons of the ciliary ganglion, and thereby aid in clarifying their role in neurotransmission. The present studies use single- and double-label immunohistochemical techniques to examine these issues in pigeons.

\section{Materials and methods}

\section{Immunohistochemical procedures}

Six months to one-year-old White Carneaux pigeons (Columbia livia) were used in the present study. Birds were deeply anesthetized with $1 \mathrm{ml}$ of $35 \%$ chloral hydrate and perfused transcardially with $6 \%$ dextran in $0.1 \mathrm{M}$ phosphate buffer (PB) (pH 7.2) followed by the paraformaldehyde-lysine-periodate (PLP) fixative of McLean and Nakane (1974). The brains were removed and stored in a solution of $20 \%$ sucrose- $10 \%$ glycerin in $0.1 \mathrm{M}$ phosphate buffer (containing $0.02 \%$ sodium azide) at $4^{\circ} \mathrm{C}$ until sectioned. The brains were sectioned frozen in the transverse plane at $40 \mu \mathrm{m}$ on a sliding microtome and processed immunohistochemically as free-floating sections. Eyes were removed from the skull and the cornea, iris, and lens dissected away. The remaining eyecups were then stored in a solution of $20 \%$ sucrose in $0.1 \mathrm{M}$ phosphate buffer at $4^{\circ} \mathrm{C}$ until sectioned at $20 \mu \mathrm{m}$ on a cryostat. Serial sections through the ciliary ganglion (which is situated temporal to the optic nerve as it leaves the eye) were collected on gelatin-coated slides and dried on a slide warmer at $37^{\circ} \mathrm{C}$. Immunohistochemical processing was carried out on these sections by incubating the sections in antisera droplets on the slide, as described previously (Erichsen et al., 1982a,b; Reiner et al., 1984). The type of immunohistochemical procedure used depended on the goal in that particular study. In the case of single-labeling studies, the immunofluorescence procedure or the peroxidase-antiperoxidase (PAP) procedure were used (Erichsen et al., 1982a; Reiner et al., 1983b, 1984; Reiner, 1987b). In order to determine whether specific pairs of neuroactive substances were co-localized, a simultaneous immunofluoresence double-labeling procedure was employed. Details of our single-labeling and double-labeling immunofluorescence procedures and our PAP procedures have been described previously (Erichsen et al., 1982a,b; Reiner et al., 1985; Reiner, 1986, 1987a,b; Reiner \& Carraway, 1987; Wessendorf \& Elde, 1985; Anderson \& Reiner, 1990a,b). In the case of studies examining the numbers of single-labeled and double-labeled peptidergic perikarya in EW, perikaryal labeling was enhanced by intraventricular injections of colchicine (200 $\mu \mathrm{g}$ in $3 \mu \mathrm{l}$ of distilled water) $24-36 \mathrm{~h}$ prior to sacrifice. The birds were deeply anesthetized with ketamine (Ketaset ${ }^{\circledR}$, $0.66 \mathrm{ml} / \mathrm{kg}$ ) and xylazine $\left(\mathrm{Gemini}^{\odot}, 0.33 \mathrm{ml} / \mathrm{kg}\right.$ ) during these 
injections and stereotaxic techniques under aseptic surgical conditions were used (Karten \& Hodos, 1967).

\section{Antisera used}

A number of different antisera were used in the present study, all of which have been shown to be highly specific for their target antigens in previous studies (with the exception of an extensive crossreactivity of the anti-LENK antisera with MENK and of the anti-MENK with LENK). The antisera were (1) a rat monoclonal antibody against substance $P$ (purchased from Accurate Chemical and Scientific Co., Westbury, NY) (Cuello et al., 1979; Reiner et al., 1984), (2) an antiserum raised in rabbit against LENK (purchased from ImmunoNuclear Corp., Stillwater, MN) (Reiner, 1987b), (3) a mouse monoclonal antibody against LENK (purchased from Accurate Chemical and Scientific Co.) (Cuello et al., 1984; Reiner, 1987b), (4) an antiserum raised in rabbit against methionine-enkephalin-Arg6- Phe7 (MERF) (generously provided by G.J. Dockray) (Williams \& Dockray, 1982,1983; Reiner, 1987b), (5) an antiserum raised in rabbit against MENK (purchased from ImmunoNuclear Corp.) (Reiner, 1987b), (6) an antiserum raised in rabbit against BAM22P (generously provided by A. Baird) (Bloch et al., 1983; Reiner, 1987 b), and (7) an antiserum raised in rabbit against chicken choline acetyltransferase (ChAT) (generously provided by Miles Epstein and June Dahl) (Johnson \& Epstein, 1986; Millar et al., 1987; Sorenson et al., 1989).

\section{Lesion studies}

In several pigeons, electrolytic lesions of $\mathrm{EW}$ were made in order to (1) confirm that EW was the source of the peptidergic terminals in the ciliary ganglion, and (2) determine if the boutonal endings arise from within a different portion of EW than the cap-like endings. To make these lesions, an insulated tungsten electrode (AM systems, $10 \mathrm{M} \Omega$ ) was lowered into EW on the left side of the brain of deeply anesthetized pigeons (Ketaset $^{\oplus}, 0.66 \mathrm{ml} / \mathrm{kg}$; and Gemini ${ }^{\oplus}, 0.33 \mathrm{ml} / \mathrm{kg}$ ), using stereotaxic techniques under aseptic surgical conditions (Karten \& Hodos, 1967). To confirm the placement of the electrode tip in $\mathrm{EW}$, a $100-\mathrm{Hz}$ pulse train $(0.5$-ms pulse duration, $20-400 \mu \mathrm{A}$ anodal current pulses) was passed between the electrode tip and a reference electrode clipped to the bird's foot (using a Grass S48 stimulator and a simulus isolation unit). Electrodes placed in EW were found to yield pupil constriction with threshold currents of $20-100 \mu \mathrm{A}$. Following the initial stereotaxic placement of the electrode, the electrode placement was altered as necessary to yield pupil constriction with these currents. Once the electrode was positioned in EW, 0.2-2 mA anodal constant current was passed for $30 \mathrm{~s}$ (using a Grass S48 stimulator and a d.c. constant current unit). Following a two-week survival period, the birds were deeply anesthetized and perfused with dextran followed by the PLP fixative, as described above. The eyes were removed immediately after perfusion with the ciliary ganglion still attached as described above and stored at $4^{\circ} \mathrm{C}$ in $25 \%$ sucrose-PB until sections through the ciliary ganglion were cut on a cryostat and collected on glass slides. The sections were then processed as described above. The brains of these animals were sectioned transversely at $40 \mu \mathrm{m}$ and every third section was stained with cresyl violet to determine the location of the lesion site. In the case of some subtotal EW lesions, an additional se- ries of sections was processed according to the PAP procedure using the anti-ChAT antibody to examine the extent of the EW lesion in more detail.

\section{HRP studies}

Finally, in several pigeons the central location of neurons projecting to the ciliary ganglion was examined by injecting horseradish peroxidase (HRP) into the ciliary ganglion and examining the distribution of retrogradely labeled neurons in the central nervous system. To make these HRP injections, the birds were deeply anesthetized as described above and the supraorbital bone dissected away under surgical aseptic conditions. The eyeball was then partially collapsed by suctioning with a 27 -gauge hypodermic needle attached to a $1-\mathrm{ml}$ syringe. The ciliary ganglion was then exposed and injected with $0.1 \mu \mathrm{l}$ of a $30 \%$ solution of HRP, using a micropipette attached to a Hamilton microsyringe. The eye reinflated within 1-2 h. Following a $24-\mathrm{h}$ survival period, the bird was deeply anesthetized and perfused transcardially with $6 \%$ dextran in phosphate buffer followed by $1 \%$ paraformaldehyde- $1.25 \%$ glutaraldehyde in $0.1 \mathrm{M}$ phosphate buffer ( $\mathrm{pH} 7.4)$. The brain was then removed, immersed in a solution of $30 \%$ sucrose in $0.1 \mathrm{M} \mathrm{PB}$, and sectioned frozen in the transverse plane at $50 \mu \mathrm{m}$ on a sliding microtome. Serial sections through the midbrain were processed for HRP using the tetramethylbenzidine (TMB) procedure (Mesulam, 1978), with the glucose-oxidase modification (Itoh et al., 1979).

\section{Results}

\section{Studies of the nucleus of Edinger-Westphal}

\section{Anatomical localization of EW: Nissl staining and ChAT immunohistochemistry}

EW is cytoarchitectonically distinct in Nissl-stained material (Figs. 1 and 2). Viewed in the transverse plane, this cell group is roughly oval in outline and is situated at the dorsolateral edge of the somatic subdivision of the oculomotor nuclear complex (OM). EW does not extend throughout the entire rostrocaudal extent of $\mathrm{OM}$, but is only found through the middle portion of OM (Fig. 3). In Nissl-stained material, two subdivisions, a medial and a lateral, can be recognized, with large neurons being more abundant in lateral EW than in medial EW (Figs. 1 and 2) (Gamlin et al., 1984). The medial subdivision extends more rostrally than the lateral, while the lateral extends more caudally. Anti-ChAT immunohistochemistry served to confirm the extent and location of OM and EW (Figs. 2 and 3). Within the somatic portions of the oculomotor nuclear complex, large cholinergic neurons were observed to be distributed in the "butterfly" shape characteristically seen in Nissl-stained material (Figs. 1-3). The neurons of OM are known to innervate four different eye muscles and the location of the pool of neurons innervating each has been identified (Brecha \& Karten, 1981; Evinger, 1988). These four pools were identifiable in the ChATlabeled material. In addition, a fifth distinct pool of motoneurons was evident at the dorsomedial edge of OM, adjacent to the medial rectus motoneurons. Although this pool of small motoneurons has not been well-studied, recent HRP studies have revealed that this pool innervates the medial rectus muscle (Erichsen \& Evinger, 1989). ChAT + neurons of EW were confined to the boundaries of EW that were observed in Nissl- 

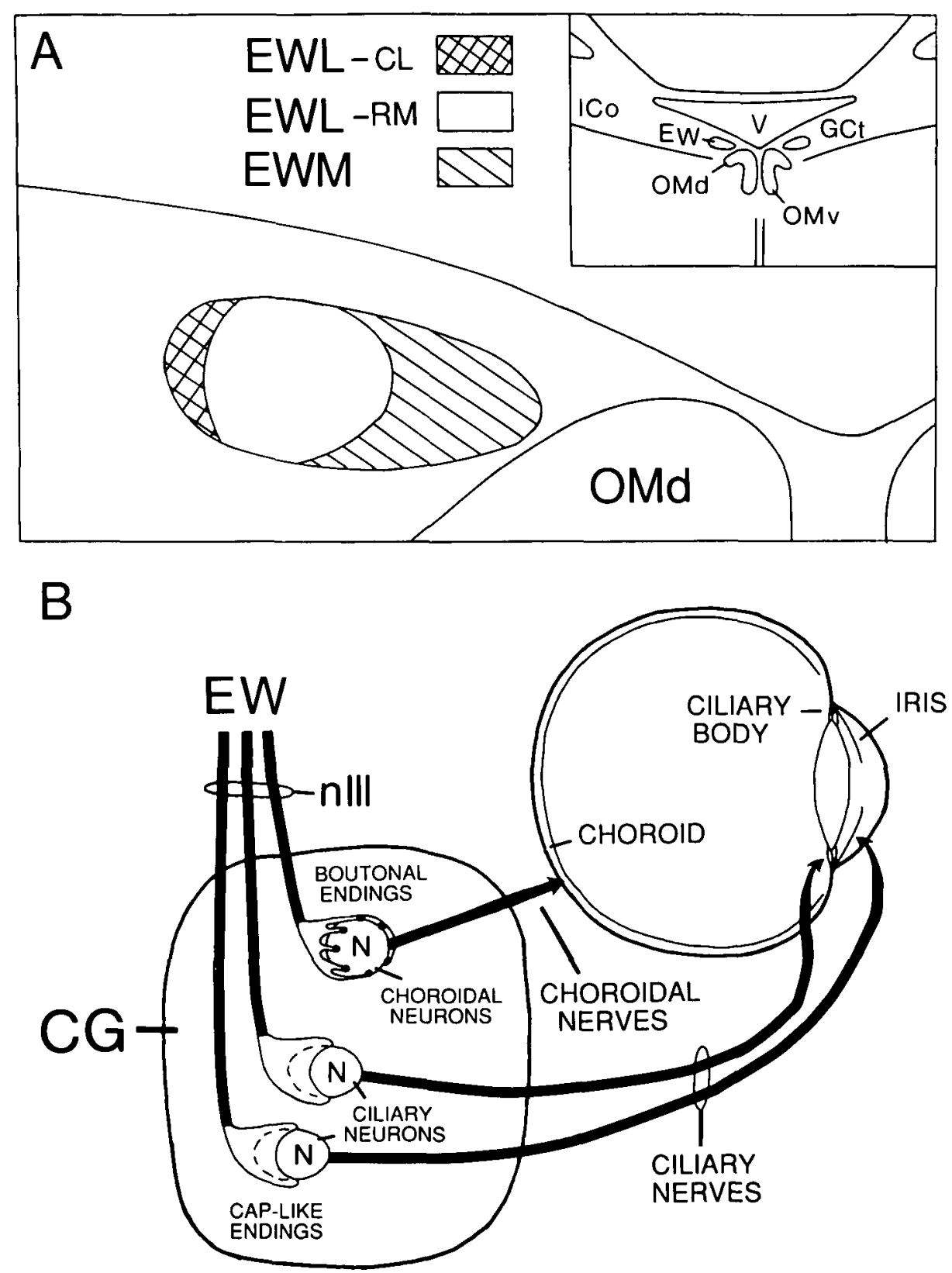

Fig. 1. A: Schematic showing the location of the nucleus of Edinger-Westphal (EW) within the midbrain (see inset) and its subdivisions, as viewed in the transverse plane. Based on cytoarchitecture, two subdivisions of EW are apparent, a medial (EWM) and a lateral (EWL). Pathway tracing studies have shown that the more caudolateral part of EWL (i.e. EWL-CL), but not the more rostromedial part of EWL (i.e. EWL-RM), is involved in the control of pupil constriction. B: Schematic illustrating the two morphologically distinct types of preganglionic endings and their target neurons in the ciliary ganglion (CG). GCt: central gray; ICo: nucleus intercollicularis; nIII: oculomotor nerve; OMd: dorsal subdivision of the oculomotor nucleus; OMv; ventral subdivision of the oculomotor nucleus; V: ventricle.

stained material. ChAT + neurons in medial EW were approximately $18-25 \mu \mathrm{m}$ in diameter, while ChAT + neurons in lateral EW were approximately $25-32 \mu \mathrm{m}$ in diameter. Counts of the number of labeled neurons in EW (adjusted by Abercrombie's correction) (Abercrombie, 1946) in one ChAT-stained series of every third section through EW indicated that about 1200 ChAT + neurons are present in EW. Of these, approximately 700 are found in the medial part of EW (EWM) and approximately 500 in the lateral part of EW (EWL) (Fig. 4). Since we observed that neurons were no more abundant in EW in Nissl- stained material than in ChAT-labeled material, it seems likely that virtually all neurons of EW are cholinergic.

\section{Peptides in EW neurons: Single-label studies}

Substance $P$ and LENK are also present in neurons of EW, as are the additional enkephalin peptides MENK, MERF, and BAM22P. These various peptides, however, are only observed in neurons of EW in colchicine-treated pigeons. Although double-label studies were not carried out to examine the co-localization of the various ENK peptides in EW neurons, it seems 

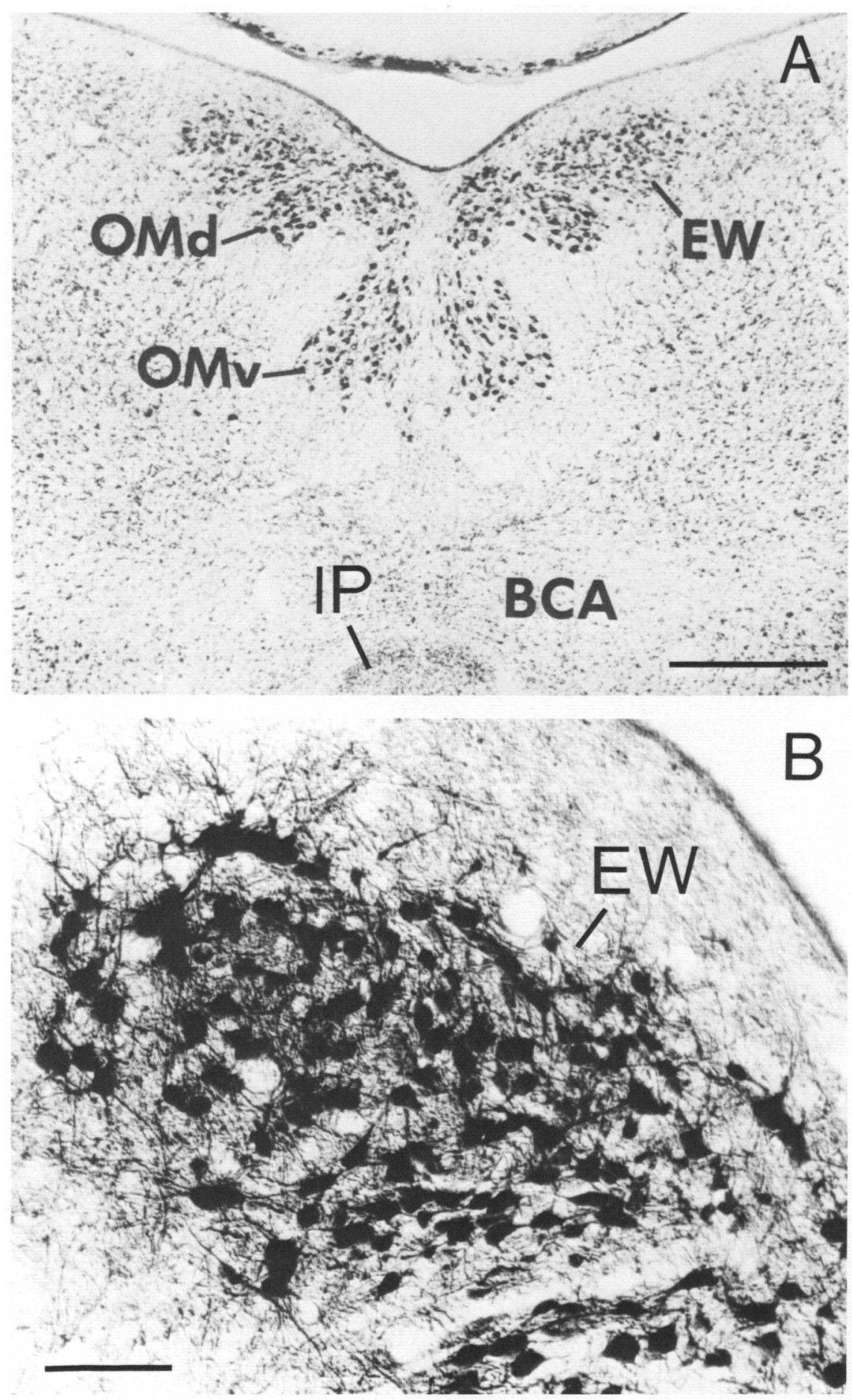

Fig. 2. A: Low-power photomicrograph of a cresyl-violet stained transverse section showing the location of $E W$ in the midbrain. Scale bar $=500 \mu \mathrm{m}$. B: Higher-power photomicrograph of EW on the left side of the brain in a section that had been labeled using the peroxidase-antiperoxidase procedure for the localization of choline acetyltransferase (ChAT). Scale bar = $100 \mu \mathrm{m}$. BCA: brachium conjunctivum ascendens; IP: interpeduncular nucleus; OMd: dorsal subdivision of oculomotor nucleus; and OMv: ventral subdivision of oculomotor nucleus.

extremely likely that they co-occur in the same neurons of EW. This conclusion is based on the results of the double-label studies in the ciliary ganglion (as described below) and on the observations in reptiles and mammals showing that LENK, MENK, MERF, and BAM22P, which all derive from proenkephalin, co-occur with one another in enkephalinergic neurons
(Khachaturian et al., 1983; Reiner, 1987b). Thus, in any subsequent discussion of LENK in perikarya of EW neurons, it should be recognized that these neurons are enkephalinergic and LENK is being used as a convenient marker to identify these neurons (Fig. 5). Since the single-label studies on ChAT localization in EW suggested that all neurons of EW are cholinergic, 


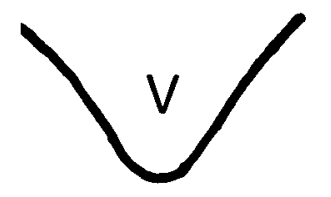

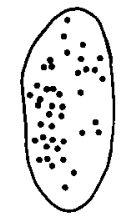

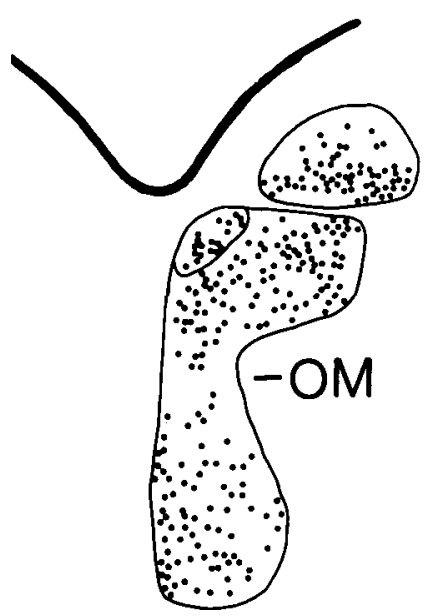

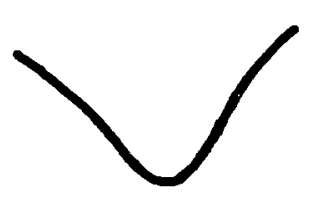
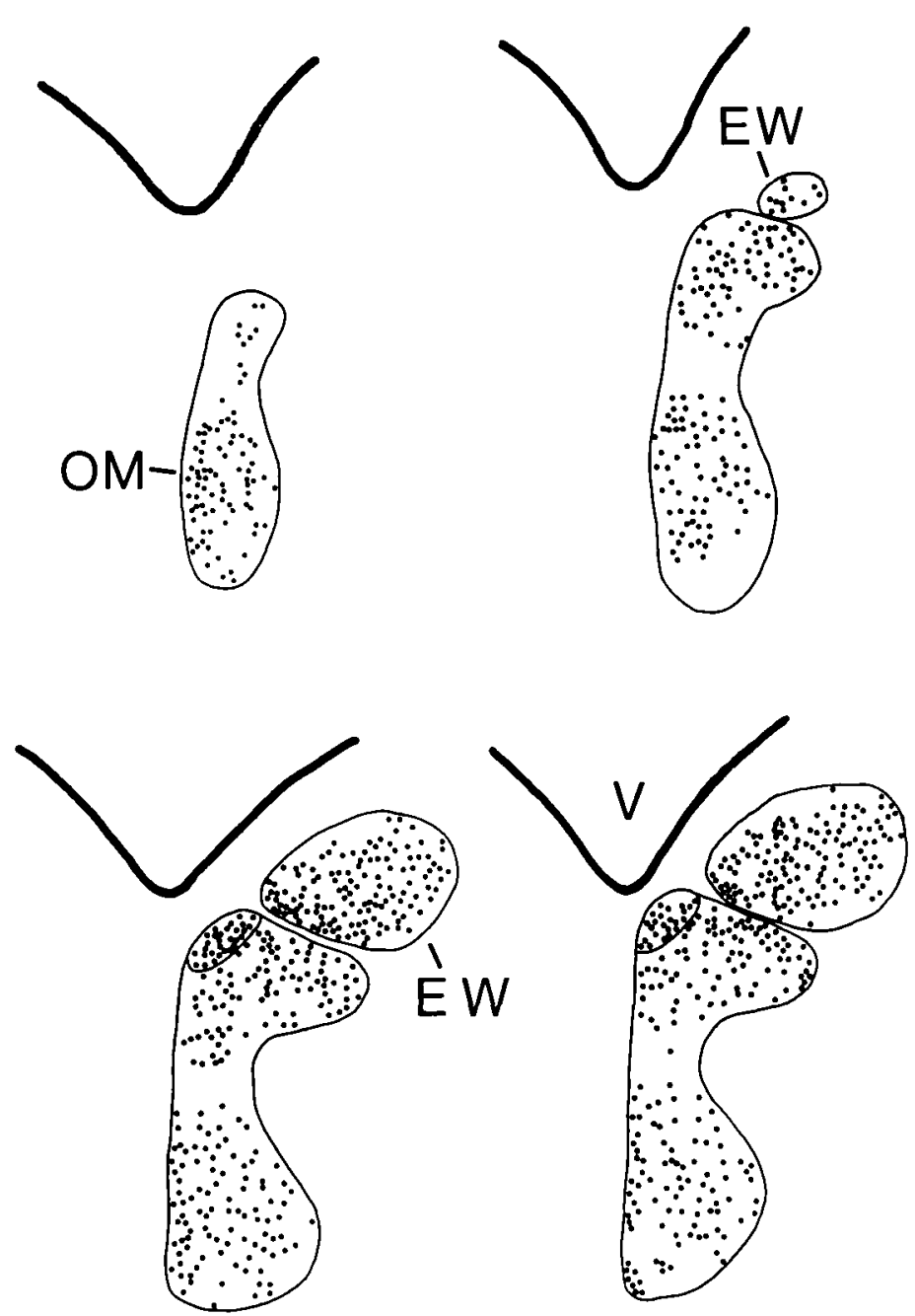

Fig. 3. Charting of every third section through the midbrain at the levels of the oculomotor nucleus (OM) and EW in a ChATlabeled series of transverse sections. These chartings illustrate the extent and location of EW with respect to OM. The sections are presented in a rostral-to-caudal sequence in a left-to-right, row-by-row sequence, with the most rostral section being shown in the upper left of the left panel and the most caudal in the lower right of the right panel. All levels shown in the left panel are rostral to those in the right panel. (Figure continued on the facing page.)

it follows that all peptidergic neurons of EW should also be cholinergic. Double-label studies were nonetheless carried out to confirm this conclusion. Additional double-label studies were carried out to examine the extent of the co-localization of SP and LENK in EW. The results of these various double-label studies are presented below.

\section{Peptides in cholinergic neurons of EW:}

\section{Double-label studies}

The studies examining SP-ChAT co-occurrence and ENKChAT co-occurrence revealed that all SP+ neurons and all LENK + neurons in EW also labeled for ChAT (Fig. 6), but many cholinergic neurons were observed that did not contain either SP or LENK. Thus, these double-label studies directly confirmed the implication of the ChAT single-label studies that all peptidergic neurons of EW are cholinergic.

The LENK+ and SP+ neurons in EW were studied in more detail in order to determine their numbers and to quantify the extent of co-localization of SP, LENK, and ChAT with one an- other in EW neurons (Fig. 4). Based on counts from a series of sections through EW from each of four different pigeons (either single- or double-labeled for SP and LENK), approximately $40 \%$ of the neurons in medial EW (EWM) and approximately $50 \%$ of the neurons in lateral EW (EWL) were found to contain either (or both) SP and LENK. Based on our single- and double-labeling studies with anti-ChAT, we assume that these figures represent the percentages of the ChAT + neurons in the two parts of EW that are also peptidergic. Among the peptidergic neurons of EWM detected immunohistochemically, nearly half were observed to contain both SP and LENK in double-label studies. Expressed as a percentage of all perikarya in EWM, 20.2\% were observed to be both SP+ and LENK+ (Figs. 4 and 7). In contrast, $11.6 \%$ of the neurons in EWM contained SP only and $10.7 \%$ contained LENK only. A slightly lower percentage of the neurons in EWL were found to contain both SP and LENK (16.2\%) than was observed in EWM. The percentages of the neurons in EWL found to contain SP only (19.2\%) and LENK only $(12.6 \%)$, however, were 

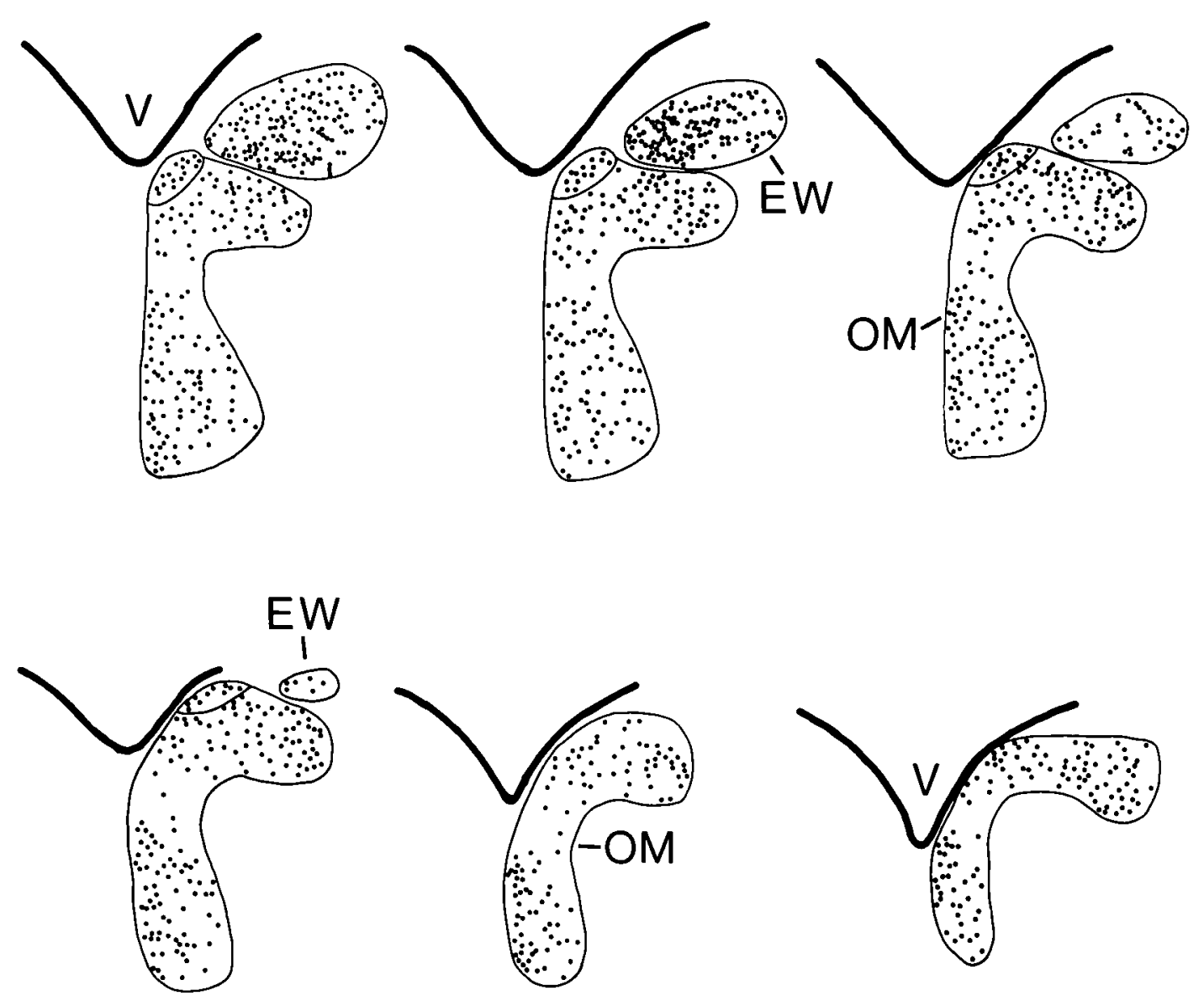

Fig. 3 continued.

slightly higher than the percentages observed in EWM for these neuronal types (Figs. 4 and 7).

Gamlin et al. (1984) have suggested that EWL consists of a caudolateral subdivision (Fig. 1) of approximately 125 neurons that is involved in pupilloconstriction and a larger, more rostromedial portion that is involved in accommodation. Although the cytoarchitectonic boundary of this pupilloconstrictive subdivision is not clear, it is interesting to note that the immunohistochemical data revealed that the relative abundances of the different types of peptidergic neurons may differ in these two subdivisions of EWL. For example, in the caudolateral part of EWL, most of the perikarya are heavily labeled for LENK (Figs. 5-7). Using the location of this distinctive group of neurons to provide a boundary between the caudolateral and rostromedial parts of EWL, we counted 100 neurons in the caudolateral part of EWL. Single- and double-label studies revealed that $74.2 \%$ of the neurons in caudolateral EWL are ENK + and approximately half of these also contained SP ( $40.5 \%$ of the total neurons in caudolateral EWL)(Fig. 7). In contrast, in the more rostromedial part of EWL, neurons containing SP are more abundant $(34.1 \%$ of the total in rostromedial EWL), and approximately one-third of these SP+ perikarya also label for LENK $(10.1 \%$ of the total in rostromedial EWL) (Fig. 7).

Projection of EW to the ciliary ganglion: HRP studies HRP injections were made into the left ciliary ganglion in three pigeons, and the location of the retrogradely labeled neu- rons in the midbrain examined using TMB histochemistry. The results showed that only neurons within the boundaries of the EW ipsilateral to the injected ciliary ganglion project to the ciliary ganglion (Figs. 8 and 9). Some HRP-labeled motoneurons were observed in $\mathrm{OM}$, but this labeling could be attributed to spread of HRP to the oculomotor muscles innervated by OM. Although we could not be certain that all neurons of EW that project to the ciliary ganglion were retrogradely labeled by these injections into the ciliary ganglion, about 800-1100 (corrected by the Abercrombie correction, Abercrombie, 1946) HRP-labeled neurons were observed in EW in the cases with the largest HRP injections into the ciliary ganglion. This number is very close to the total number of EW neurons estimated from the ChAT-labeled material. Thus, these results indicate that all or virtually all neurons of EW project to the ciliary ganglion. Furthermore, these results suggest that the projection to the ciliary ganglion arises only from EW.

Projection of EW to the ciliary ganglion: Lesion studies

The lesions of EW were grouped into three categories: (1) total unilateral EW lesions; (2) lesions that damaged much of EW but spared all or parts of EWL; and (3) lesions that damaged much of EW but spared all or parts of EWM. The ciliary ganglion on the nonlesioned side of the brain served as the control. Total unilateral EW lesions were found to completely eliminate all SP+ and LENK+ preganglionic terminals in the ciliary ganglion, thereby demonstrating that the SP+ and LENK+ terminals in the ciliary ganglion do arise from EW (Fig. 10). 

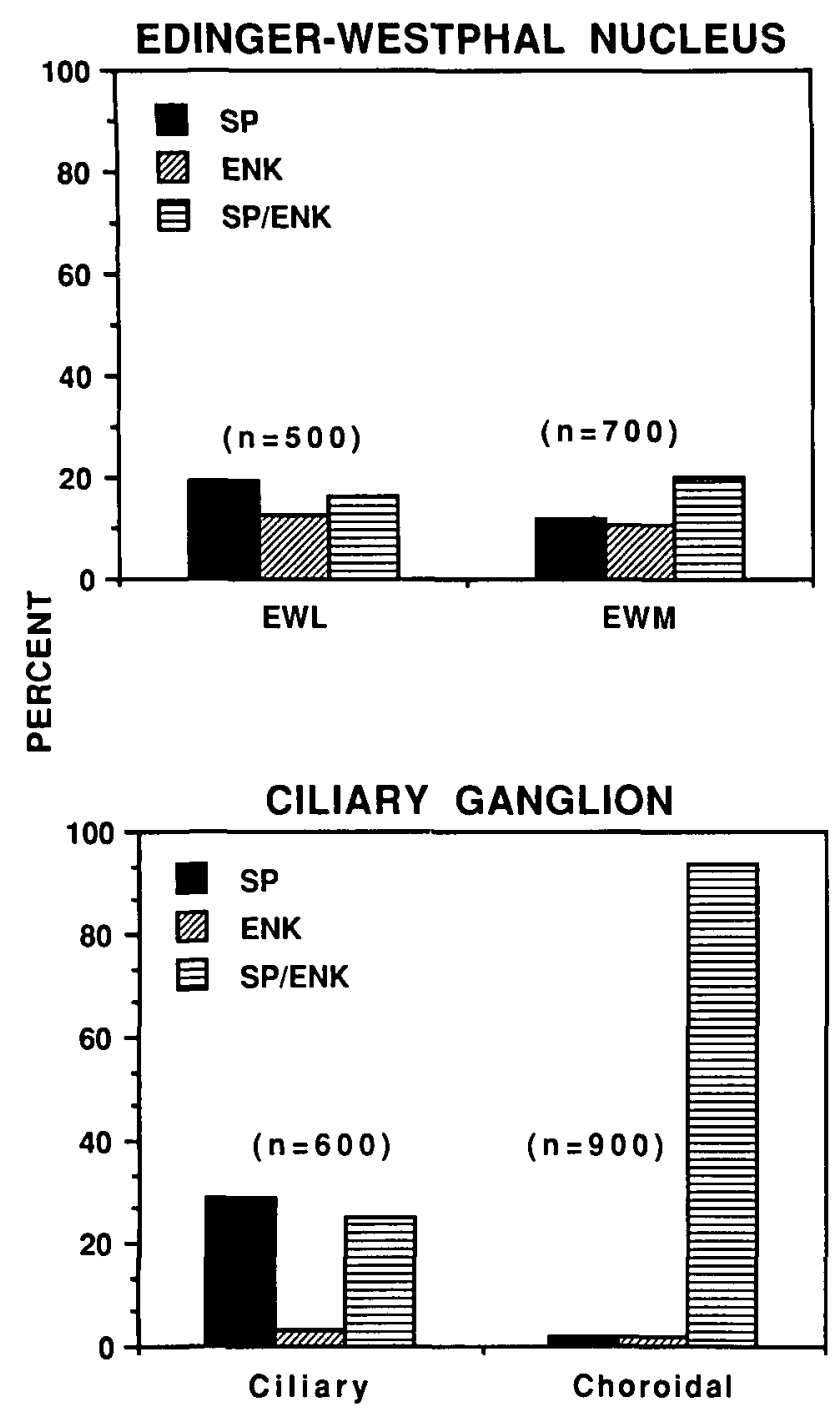

Fig. 4. Graphic representation of the percentage of EWL and EWM neurons that contain SP, ENK, or SP and ENK, and the percentage of ciliary and choroidal neurons in the ciliary ganglion that receive preganglionic endings containing SP, ENK or SP and ENK. See text for the precise percentages.

Following lesions that destroyed EWM but spared all or parts of EWL, only cap-like endings labeled for SP or LENK were observed in the ipsilateral ciliary ganglion (Fig. 11). In contrast, following lesions that destroyed EWL but spared all or parts of EWM, only boutonal endings labeled for SP or LENK were observed in the ciliary ganglion (Fig. 11). Thus, boutonal endings appear to arise from EWM, while cap-like endings appear to arise from EWL.

\section{Summary of EW studies}

EW is a discrete nucleus in pigeons, all of whose neurons appear to be cholinergic and all of whose neurons appear to project to the ciliary ganglion. Furthermore, only neurons of EW appear to give rise to preganglionic projections to ciliary ganglion neurons. Many (but not all) neurons of EW appear to also contain either or both SP and LENK. All SP+ or LENK+ neurons in EW are cholinergic, but not all cholinergic neurons appear to be SP+ or LENK+. In EWM, many (20\%) of the neurons were observed to contain both SP and LENK, while many additional neurons of EWM were observed to contain only SP or LENK, or neither. For EWL, the results were similar, except that a slightly lower percentage of neurons was observed to be double-labeled for SP and LENK and slightly higher percentages were observed to be single-labeled for SP or LENK. Whether the neurons observed to contain only ChAT are in actuality devoid of SP and/or LENK cannot be determined with certainty from these data alone, because it is possible that SP and/or LENK are present in these neurons in amounts below the levels detectable with immunohistochemistry. For a similar reason, it cannot be certain that EW neurons observed to label for ChAT and only one neuropeptide contained only one neuropeptide. The results of the studies on the terminals of the EW neurons in the ciliary ganglion, in fact, suggest that our immunohistochemical studies of EW may underestimate the percentage of EW perikarya that are peptidergic. The results for the studies of the perikaryal labeling in EW are summarized in Fig. 4. The lesion studies suggest that all peptidergic endings in the ciliary ganglion arise from EW, with boutonal endings arising from EWM and cap-like endings from EWL.

\section{Studies of the ciliary ganglion}

\section{SP and LENK in preganglionic terminals}

Many preganglionic terminals of the boutonal type and many preganglionic terminals of the cap-like type were found to be labeled for SP or LENK (Figs. 10 and 11). Consistent with the descriptions of the location of ciliary and choroidal neurons (Pilar et al., 1980), boutonal endings on choroidal neurons were observed to be highly abundant in more posterior and inferior portions of the ciliary ganglion (as the ganglion is oriented in situ), while the cap-like endings on ciliary neurons were more abundant in rostral and superior portions of the ciliary ganglion. Double-labeled studies were carried out to determine the numbers of SP+, LENK +, and SP+/LENK + terminals of each type. To aid in determining the relative abundance of these different types of terminals in the ciliary ganglion, the number of ciliary and choroidal neurons were counted in every fourth section in a ChAT-stained series of sections through the ciliary ganglion. Both types of neurons and their preganglionic inputs were labeled by the ChAT antibodies, although ciliary neurons and their cap-like endings were more intensely labeled than were choroidal neurons and their boutonal endings (Fig. 12). The fact that the anti-ChAT antiserum labeled the terminals on the two different types of ciliary ganglion neurons allowed us to distinguish unambiguously between these two types of neurons. On this basis, approximately 900 choroidal neurons and approximately 600 ciliary neurons were counted in the ciliary ganglion (corrected by Abercrombie's correction, Abercrombie, 1946) (Fig. 4). Based on counts from three separate series of sections through the ciliary ganglion, the vast majority $(93.6 \%)$ of the choroidal neurons were observed to receive boutonal endings that labeled for both SP and LENK (Figs. 4 and 13). Only $2.1 \%$ of the choroidal neurons received inputs that were all labeled for SP only, while $1.6 \%$ of the choroidal neurons received inputs that all labeled for LENK only. Among the ciliary neurons, $24.7 \%$ received inputs containing SP and LENK, $29.0 \%$ received endings labeled for SP only and $3.1 \%$ received endings labeled for LENK only (Figs. 4 and 13). The cap-like endings labeled for SP only tended to be more lightly labeled for SP than those containing both SP and LENK. For both boutonal and cap-like endings, there was no obvious regional clustering 

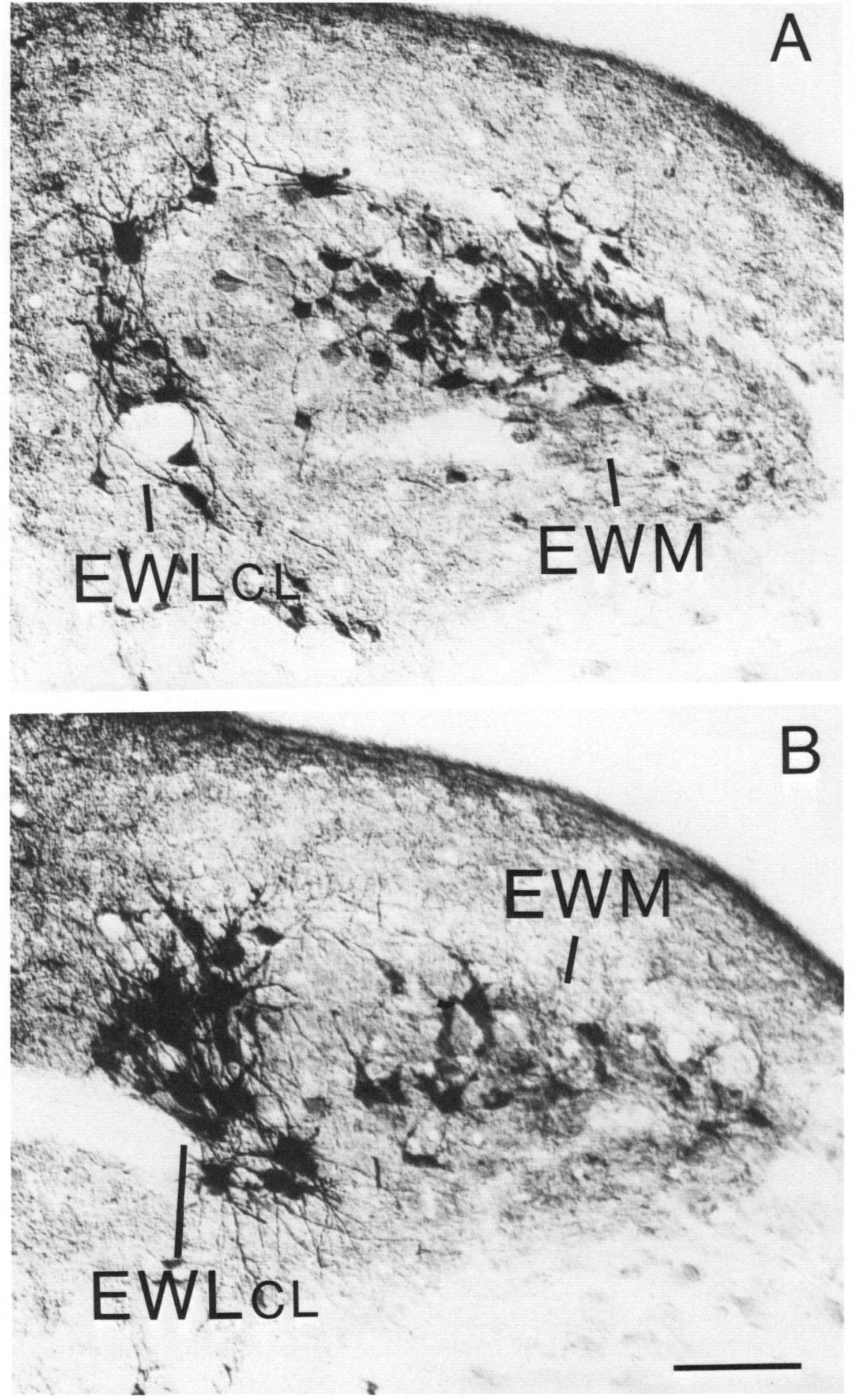

Fig. 5. Photomicrographs of ENK + perikarya in EW at rostral (A) and caudal (B) levels of EW, as seen in the transverse plane using differential interference contrast optics. Note the conspicuous cluster of intensely ENK+ perikarya in the caudolateral part of EWL. Scale bar in $B=100 \mu \mathrm{m} ; A$ is of the same magnification as in $B$.

in the distributions of SP/LENK, SP-only, or LENK-only terminals. Based on the results of our studies on EW, we assume that all preganglionic terminals in the ciliary ganglion, regardless of whether they are peptidergic or not, are also cholinergic.

\section{Other peptides in preganglionic terminals}

Since studies in birds, reptiles, and mammals (Lindberg, 1986, Reiner, 1987b) have shown that a variety of enkephalin peptides (including MENK, MERF, and BAM22P) derive from the same precursor (i.e. proenkephalin) that gives rise to LENK, the presence of MENK, MERF, and BAM22P in terminals in the ciliary ganglion was explored. All three were observed in endings on the vast majority of the choroidal neurons and in many cap-like endings. Double-label studies showed that essentially all preganglionic endings in the ciliary ganglion that contain LENK also contain MERF and BAM22P. The cooccurrence of MENK and LENK in the endings in the ciliary ganglion could not be explored directly because the anti-LENK 

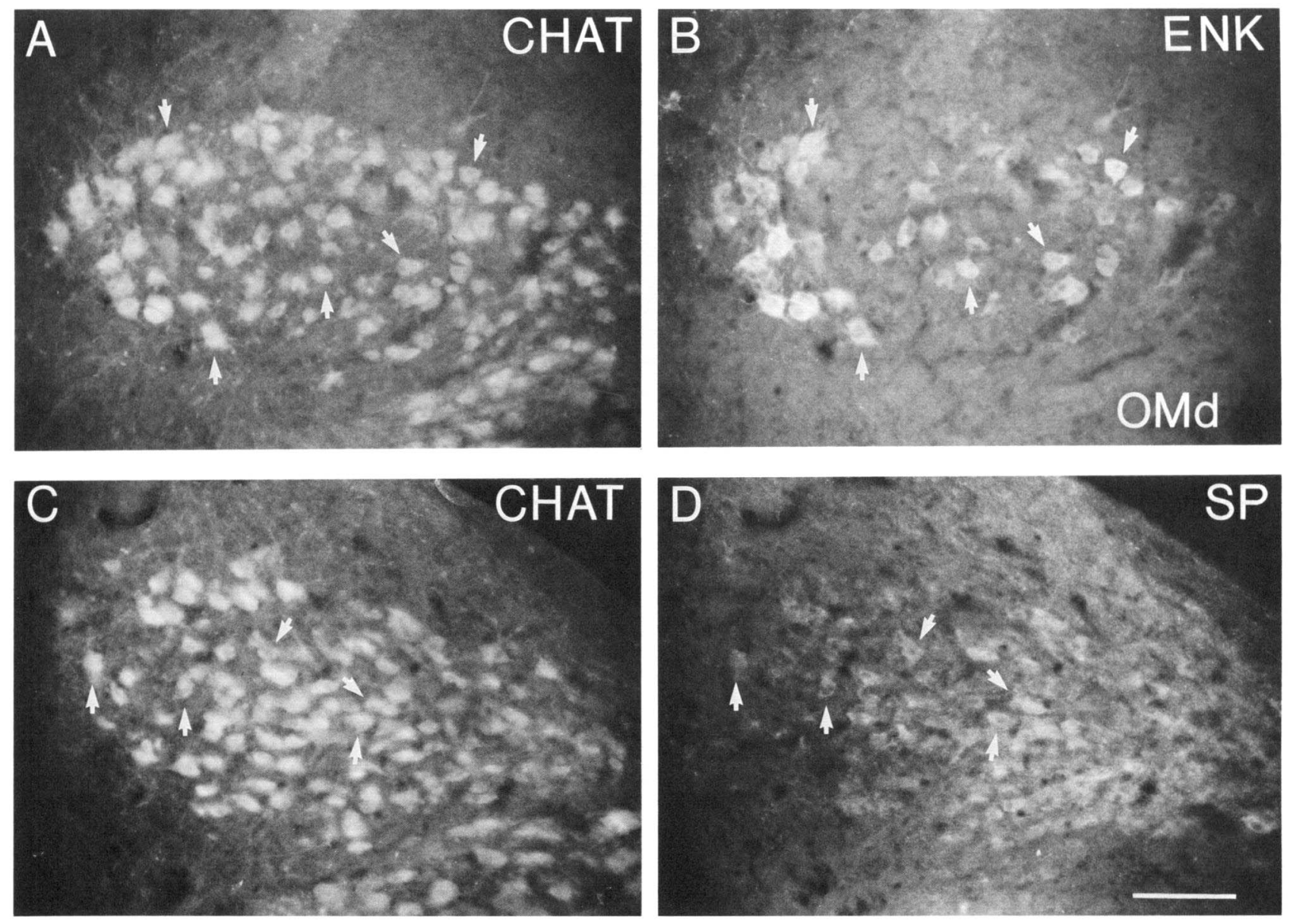

Fig. 6. Pairs of photomicrographs illustrating the presence of ENK in ChAT+ EW neurons (A and B) and the presence of SP in ChAT+EW neurons (C and D), as revealed using immunofluorescence double-labeling procedures. Photomicrographs $A$ and $B$ show the same field of view from a single section double-labeled for ChAT and ENK, with the arrows in A indicating some of the ChAT + neurons that also contain ENK, as shown in B. Photomicrographs C and D show a single field of view from a section that had been double-labeled for ChAT and SP, with the arrows in C indicating some of the ChAT+ perikarya that also contain SP, as shown in D. Scale bar in $\mathrm{D}=100 \mu \mathrm{m} ; \mathrm{A}-\mathrm{C}$ are of the same magnification as in D.

antiserum used in the double-label studies crossreacts extensively with MENK. Single-labeling studies using the anti-MENK (which is largely specific for MENK) showed that the distribution and abundance of labeled terminals in the ciliary ganglion was the same with this antiserum as with the anti-LENK. These results and the fact that LENK and MENK arise from the same precursor strongly suggest that MENK and LENK are in the same preganglionic terminals. Thus, LENK, MENK, MERF, and BAM22P all appear to be present in the same endings in the ciliary ganglion, endings which in many cases also contain SP and, in all cases, contain acetylcholine.

\section{Summary of ciliary ganglion studies}

These studies show that nearly all choroidal neurons receive boutonal endings that co-contain SP, acetylcholine, and a variety of enkephalin peptides. Ciliary neurons appear to consist of at least three major groups that are comparable in relative abundance: (1) those that receive cap-like endings that are exclusively cholinergic; (2) those that receive cap-like endings that co-contain acetylcholine, SP, and a variety of enkephalin peptides; and (3) those that receive cap-like endings that co-contain acetylcholine and SP. In addition, a relatively small group of cil- iary neurons receives input from cap-like endings co-containing acetylcholine and a variety of enkephalin peptides.

\section{Discussion}

The present results support several conclusions about the neurotransmitter and neuropeptide organization of the nucleus of Edinger-Westphal and its projection to the ciliary ganglion (Fig. 14): (1) All neurons that project to the ciliary ganglion are cholinergic and are localized to a discrete nucleus termed EW that is located dorsolateral to the somatic subdivisions of the oculomotor nuclear complex; (2) Neurons of EWM are the source of the boutonal preganglionic endings on the choroidal neurons of the ciliary ganglion, while neurons of EWL are the source of the cap-like endings on ciliary neurons of the ciliary ganglion; (3) Nearly half (if not more) of the neurons of EWM and their endings in the ciliary ganglion co-contain SP, LENK, and acetylcholine; (4) A high percentage of the neurons of EWL cocontain SP, LENK, and acetylcholine; (5) A high percentage of the neurons in EWL co-contain SP and acetylcholine, particularly in the rostromedial accommodative subdivision of EWL; (6) The majority of the neurons in the caudolateral, pupillocon- 

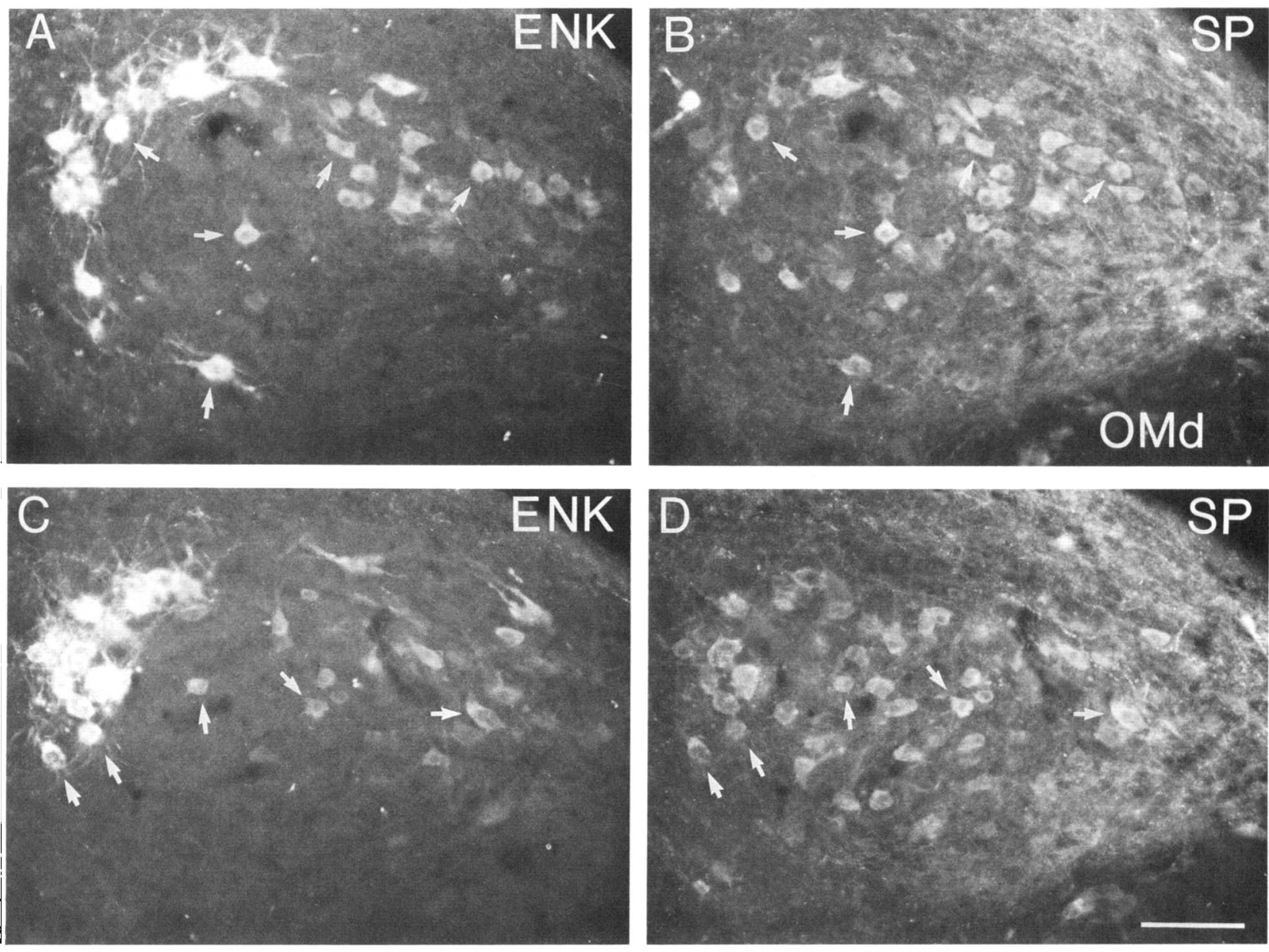

Fig. 7. Two pairs of photomicrographs ( $A$ and $B ; C$ and $D)$ illustrating the presence of SP in ENK+ EW neurons (A and B), as revealed using immunofluorescence double-labeling procedures. Photomicrographs $A$ and B show the same field of view from a single section double-labeled for SP and ENK, with the arrows in A indicating some of the ENK+ neurons that also contain $S P$, as shown in $B$. Photomicrographs $C$ and $D$ show a single field of view from a section that had been double-labeled for ENK and SP, with the arrows in C indicating some of the ENK + perikarya that also contain SP, as shown in D. Note, however, that not all labeled perikarya in the A-B pair or in the C-D pair contain both SP and ENK. Scale bar in D $=100 \mu \mathrm{m} ; A-C$ are of the same magnification as in $D$.

strictive part of EWL co-contain SP, LENK, and acetylcholine; and (7) Perikarya in EW and their preganglionic terminals in the ciliary ganglion that contain LENK also contain the additional enkephalin peptides MENK, MERF, and BAM22P.

\section{Anatomical implications}

\section{Nucleus of EW and its subdivisions}

The present studies have confirmed the previous suggestions that the nucleus of Edinger-Westphal (also called the accessory oculomotor nucleus in birds) is the exclusive source of the preganglionic input to the ciliary ganglion and projects only to the ciliary ganglion in birds (Cowan \& Wenger, 1968; Narayanan \& Nayananan, 1976; Lyman \& Mugnaini, 1980; Cabot et al., 1982; Reiner et al., 1983a). The present single- and double-label immunohistochemical studies also indicate that all peptidergic endings in the ciliary ganglion are also cholinergic and arise from EW. These results seemed likely, based on previous findings, but had not been directly demonstrated (Hess, 1965; Landmesser \& Pilar, 1970, 1978; Erichsen et al., 1982a,b; Pi- lar \& Tuttle, 1982; Reiner et al., 1983a; Sorenson et al., 1989). Finally, the present results support the conclusion that the two types of preganglionic terminals in the avian ciliary ganglion arise from different parts of EW, with boutonal endings on choroidal neurons arising from neurons of EWM and cap-like endings on ciliary neurons arising from neurons of EWL. This idea was first suggested by Gamlin et al. (1982) and Reiner et al. (1983a), and has since been supported by additional lines of evidence. First, Gamlin et al. (1984) demonstrated that the preganglionic neurons of EW that control the pupillary light reflex (and therefore give rise to cap-like endings on ciliary neurons innervating the sphincter muscle of the pupil) are located in caudolateral EWL (Fig. 14). Later, Erichsen and Evinger (1985) using retrograde transneuronal labeling with wheatgerm agglutinin (WGA) showed that neurons of EWL innervate the neurons of the ciliary ganglion that innervate the sphincter muscle of the pupil or ciliary body (i.e. ciliary neurons). Finally, Reiner et al. (1990) have recently shown using physiological techniques that the neurons of EWM control choroidal blood flow, but not pupil diameter or lens shape. Thus, the evidence is very strong 

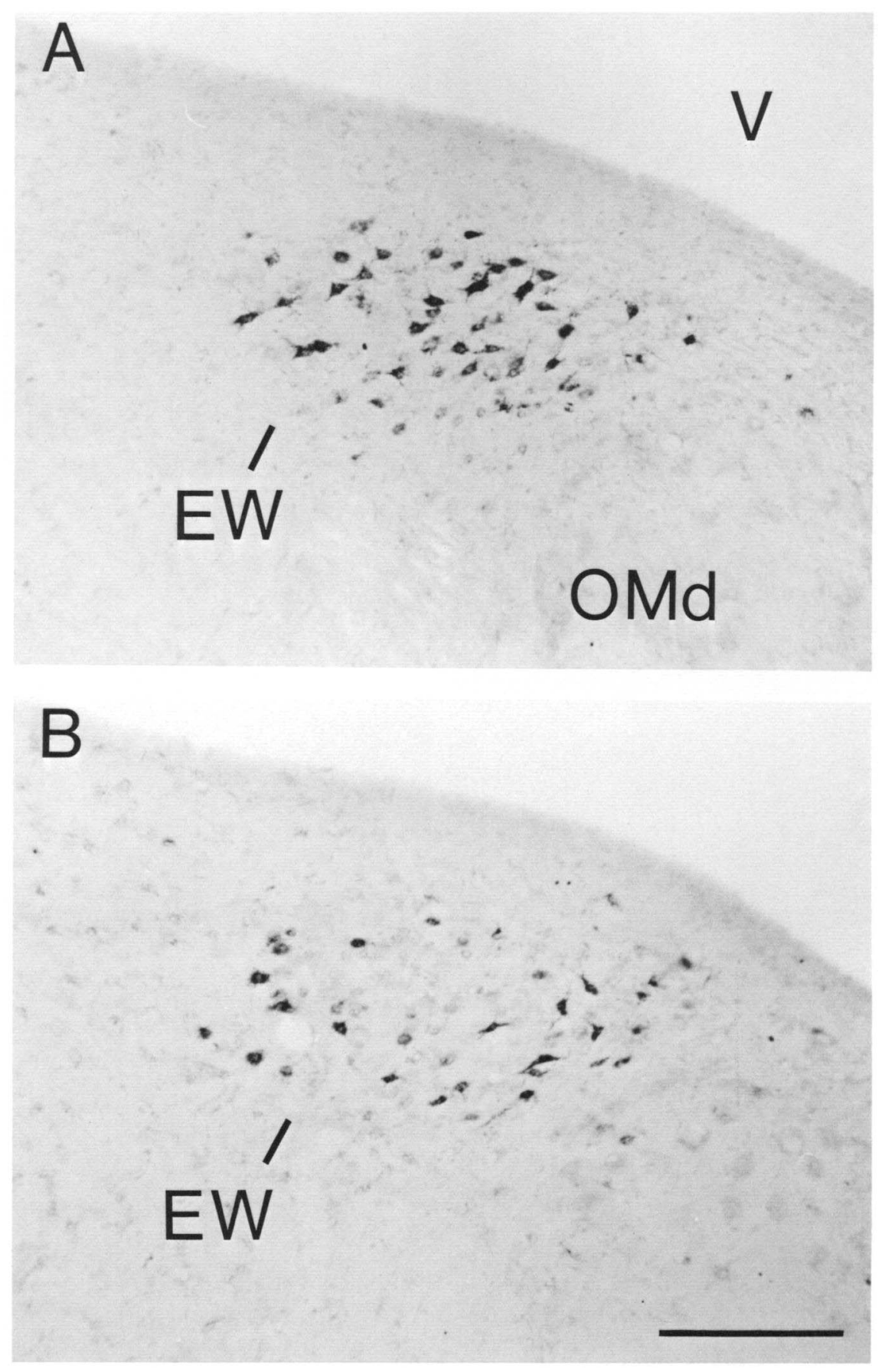

Fig. 8. HRP-labeled neurons in EW after a large HRP injection into the ipsilateral ciliary ganglion. EW is shown at a rostral (A) and caudal (B) level. Scale bar in $B=200 \mu \mathrm{m}$; A is of the same magnification as in B.

that EWM gives rise to the boutonal endings on choroidal neurons and EWL gives rise to the cap-like endings on ciliary neurons. Furthermore, the evidence also supports the conclusion that neurons in rostromedial EWL give rise to cap-like endings that end on those ciliary neurons that innervate the ciliary body, and neurons in caudolateral EWL end on those ciliary neurons that innervate the sphincter muscle of the iris.

\section{Co-localization of neuropeptides and acetylcholine in EW and the ciliary ganglion}

The present results show that nearly all choroidal neurons in the ciliary ganglion receive boutonal endings that co-contain
SP, LENK, and acetylcholine, while the majority of the ciliary neurons receive a cap-like ending that contains acetylcholine and a neuropeptide (either SP and/or LENK). Although the percentages of the neurons receiving the different types of neuropeptide-containing preganglionic endings in the ciliary ganglion support this conclusion, the percentages of the different types of peptidergic neurons in EWM and EWL are seemingly inconsistent with such a conclusion. For example, $94 \%$ of the choroidal neurons receive boutonal endings that co-contain SP, LENK, and acetylcholine, while only approximately $20 \%$ of the neurons of EWM were observed to co-contain these substances. Similarly, the percentage of perikarya in EWL labeled for both 

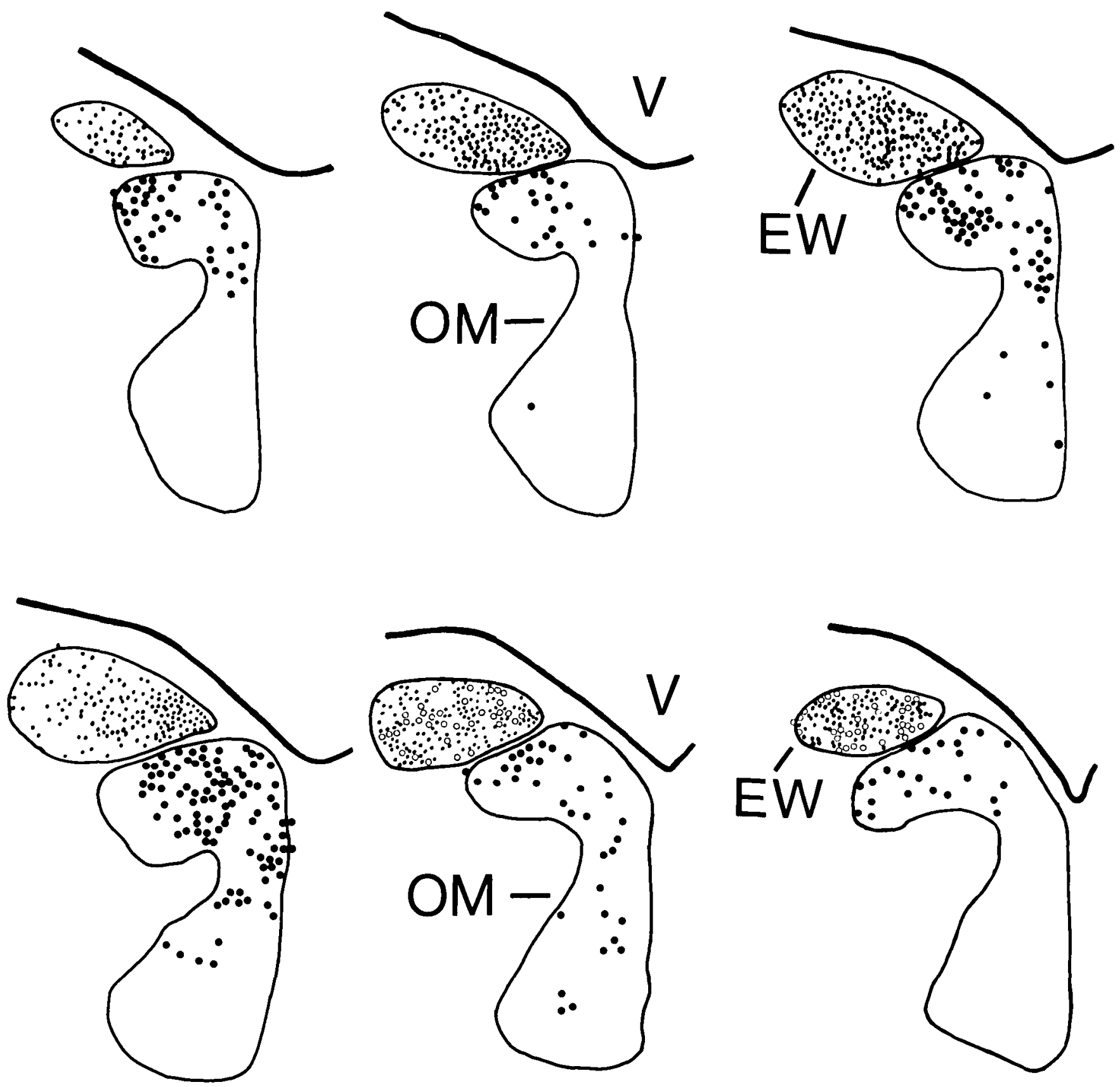

Fig. 9. Charting of every second section showing HRP-labeled cells in EW and OM after a large HRP injection in the ipsilateral ciliary ganglion that labeled $89 \%$ of the neurons in EW. The chartings are presented in a rostral-to-caudal left-to-right, rowby-row sequence. Each dot in EW represents a labeled cell, while open circles are unlabeled cells. Also shown, by large dots, is the location of HRP-labeled perikarya in OM, which were retrogradely labeled due to leakage of HRP from the ciliary ganglion to nearby extraocular muscles.

SP and LENK $(16 \%)$ is less than the percentage of the ciliary neurons in the ciliary ganglion that receive cap-like endings containing both SP and LENK (25\%). Furthermore, the percentage of SP-only (19\%) perikarya in EWL is also less than the percentage of ciliary neurons receiving input from cap-like endings containing only SP $(30 \%)$. Whether the neurons of EW that did not label for SP and/or LENK are in actuality devoid of SP and/or LENK cannot be determined, however, with certainty from the data on EW alone, because it is possible that SP and/or LENK are present in these neurons in amounts below the levels detectable with immunohistochemistry. In fact, this appears to be a plausible interpretation of at least part of the disparity between the prevalence of peptides in EW neurons as compared to the prevalence of the same peptides in preganglionic endings. For example, SP and ENK were observed to cooccur in all boutonal endings on the choroidal neurons receiving input from SP+/ENK+ boutonal endings. This would not be the case if many EW neurons, in fact, contained either SP only or ENK only. It is possible, however, that innervation of multiple ciliary ganglion neurons by individual peptidergic neurons of EW also contributed to the disparity. For example, each choroidal neuron is thought to receive input from two or more EW 


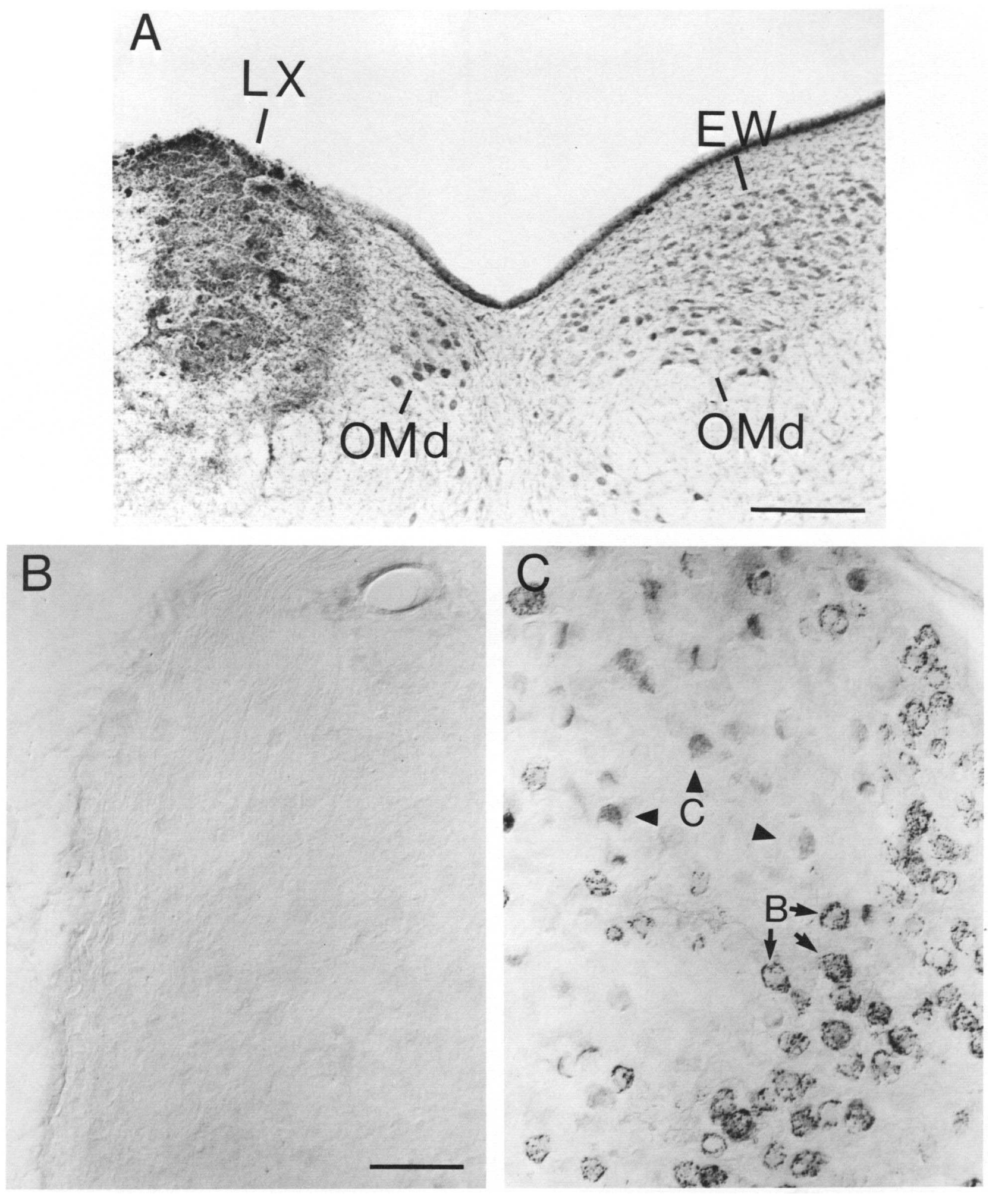

Fig. 10. Photomicrograph A shows the oculomotor region in a bird with a complete left EW lesion (LX), with intact right EW. Photomicrograph B shows a representative section through the ipsilateral ciliary ganglion of this bird that had been labeled immunohistochemically for the presence of SP. Note that no labeled boutonal or cap-like endings are evident. In contrast, numerous SP-labeled boutonal endings (some of which are indicated by the arrows and the letter "B") and cap-like endings (some of which are indicated by the arrowheads and the letter " $C$ ") were observed in the right ciliary ganglion of this bird, as shown in photomicrograph C. Scale bar in A $=300 \mu \mathrm{m}$; scale bar in $B=100 \mu \mathrm{m} ; C$ is of the same magnification as in $B$. 

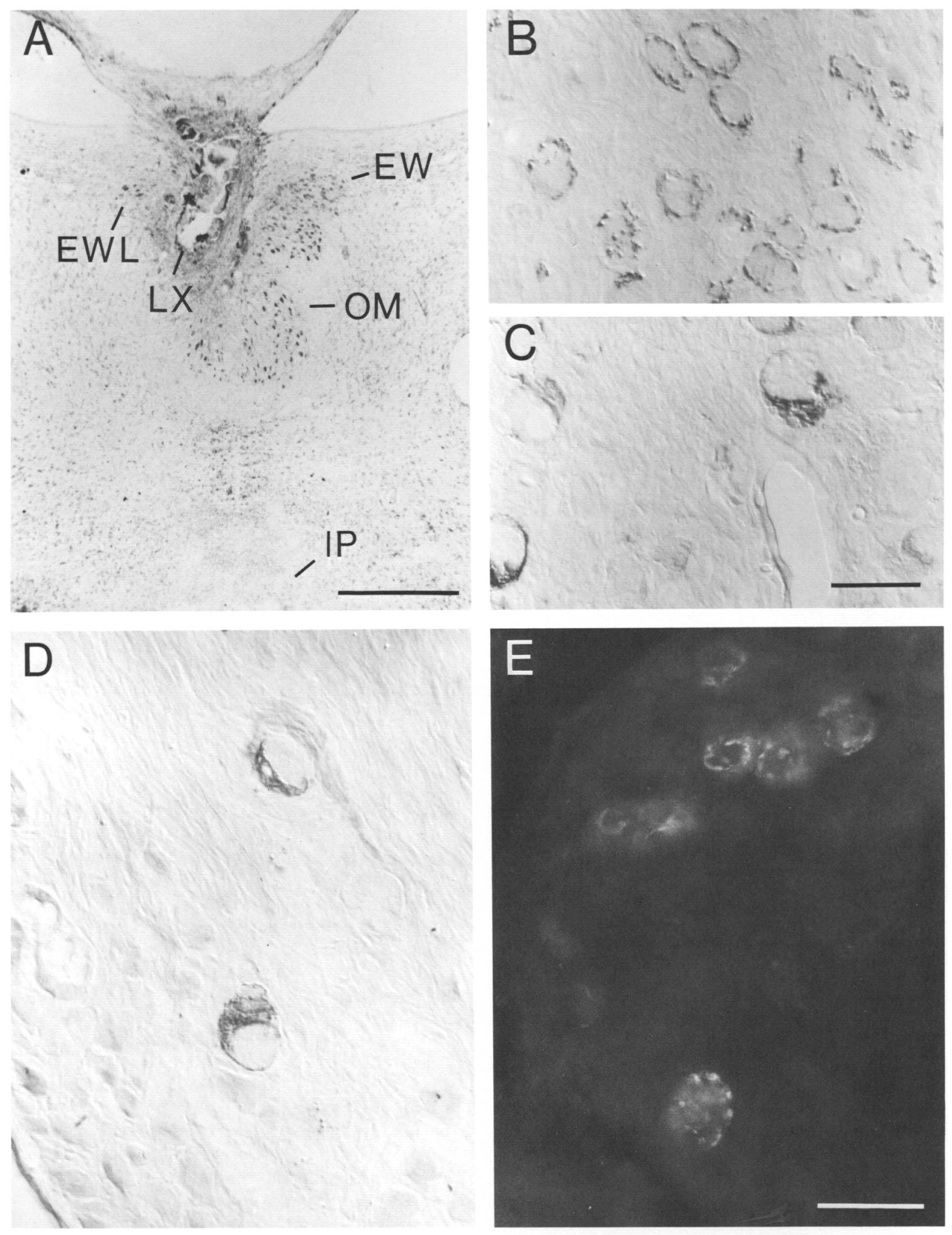

Fig. 11. Photomicrograph A shows the oculomotor region in a bird with a lesion (LX) of the left EW that spared part of EWL. Photomicrographs B and C show normal boutonal and cap-like endings in the right ciliary ganglion, as labeled immunohistochemically for SP. Photomicrograph D shows that cap-like endings, but not boutonal endings (as visualized by immunohistochemical labeling for SP), were observed in the left ciliary ganglion of the bird whose lesion is shown in photomicrograph $A$. Photomicrograph E shows a section through the left ciliary ganglion from a bird that had received a lesion of the left EW that destroyed all of EWL, but spared part of EWM. Note that in the ispilateral ciliary ganglion of this bird, some boutonal endings but no cap-like endings remained, as visualized using immunofluorescence labeling for SP. Scale bars in $A=1 \mathrm{~mm} ; \mathrm{B}, \mathrm{D}$ are of the same magnification as in $\mathrm{C}$; Scale bar in $\mathrm{C}, \mathrm{E}=50 \mu \mathrm{m}$. 

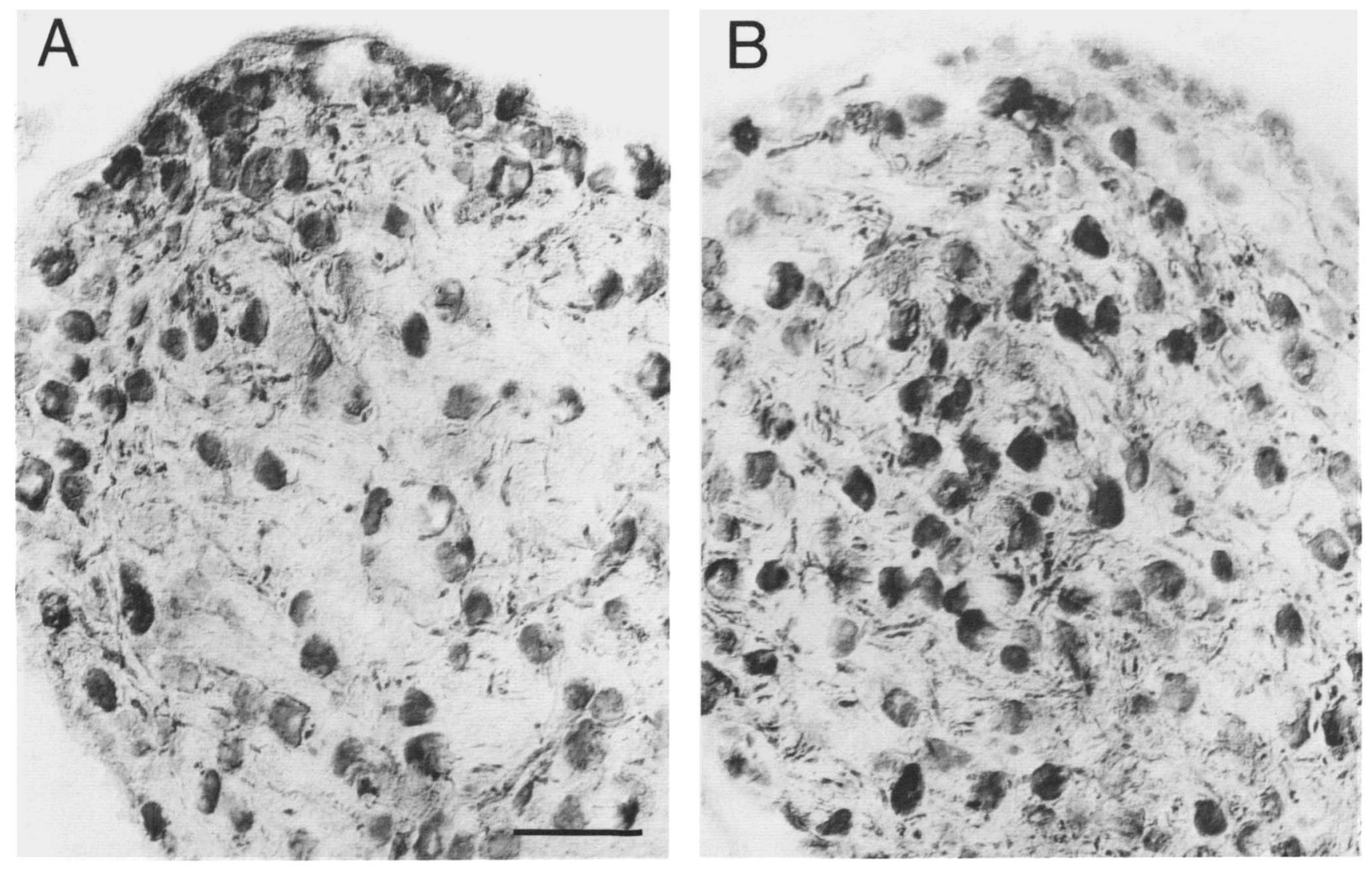

Fig. 12. Photomicrographs A and B show two sections through the ciliary ganglion that had been labeled immunohistochemically for ChAT. Note that numerous neurons in the ganglion are labeled, with the ciliary neurons and their cap-like endings tending to be more intensely labeled than the choroidal neurons and their boutonal endings. Scale bar in $A=100 \mu \mathrm{m} ; \mathrm{B}$ is of the same magnification as in $\mathbf{A}$.

neurons (Dryer \& Chiappinelli, 1985b). If this is the case for all $\mathrm{SP}+/ \mathrm{LENK}+/ \mathrm{ChAT}+\mathrm{EWM}$ neurons (which we found made up $20 \%$ of all EWM neurons) and each of these randomly innervates two to five choroidal neurons, it would be expected that the vast majority of choroidal neurons would receive $\mathrm{SP}+/$ LENK +/ChAT + endings, as we did in fact observe.

\section{Cell counts}

The numbers of EW neurons and ciliary ganglion neurons estimated in the present study are somewhat lower than those estimated in previous studies in birds. We observed a total of approximately 1200 neurons in EW, with about 700 in EWM and approximately 500 in EWL. Previously, Gamlin et al. (1984) reported about 1200 EWM neurons and 500 EWL neurons in pigeons. Similarly, our results for the number of neurons in EWL controlling pupil constriction, i.e. 100, are also very similar though slightly less than those of Gamlin et al. (1984), i.e. 125. These discrepancies might stem from several methodological differences between the two studies. Our counts were based on a series of ChAT-stained sections through EW, and we used Abercrombie's correction (Abercrombie, 1946). Furthermore, our boundary between EWM and EWL was based on an adjacent series of sections that had been labeled for SP (SP+ fiber levels are much higher in EWM than EWL) (Gamlin et al., 1982). In the Gamlin et al. (1984) study, a series of Nissl-stained sections was used, the EWM-EWL boundary was based on cytoarchitectural considerations and the Aber- crombie correction was not used. If Abercrombie's correction is applied to the total number of EW neurons reported by Gamlin et al. (which yields slightly over 1100 neurons in EW), their counts are virtually identical to ours. We therefore believe our current estimates to be accurate.

Our estimates for the number of neurons in the ciliary ganglion, however, are strikingly lower than those of previous studies in chickens, turkeys, and pigeons (Pilar \& Tuttle, 1982; Terzuolo, 1951; Landmesser \& Pilar, 1974; Pilar et al., 1980; Marwitt et al., 1971). In chickens, approximately 3800 ciliary ganglion cells (with choroidal to ciliary neurons being present in a 3:2 ratio) have been reported to be present (Landmesser \& Pilar, 1974; Pilar et al., 1980), while in turkeys approximately 6000 neurons have been reported (Terzuolo, 1951). It is possible that the differences between the studies in chickens and turkeys and our study in pigeons, in which we found only about 1500 neurons in the ciliary ganglion, stem from species differences. Since turkeys and chickens are both larger as adults than pigeons, it might be expected that their ganglia contain more neurons (Armstrong, 1982; Wikler et al., 1989). It is interesting to note, however, that the ratio of choroidal to ciliary neurons in our study $(3: 2)$ is identical to that reported for chickens. Our results, however, also differ from those in the only previous study on the number of pigeon ciliary ganglion neurons, which reported a total of approximately 6000 cells, with a oneto-one choroidal-ciliary ratio (Marwitt et al., 1971). In the Marwitt et al. study, however, the ganglion cells were not themselves 

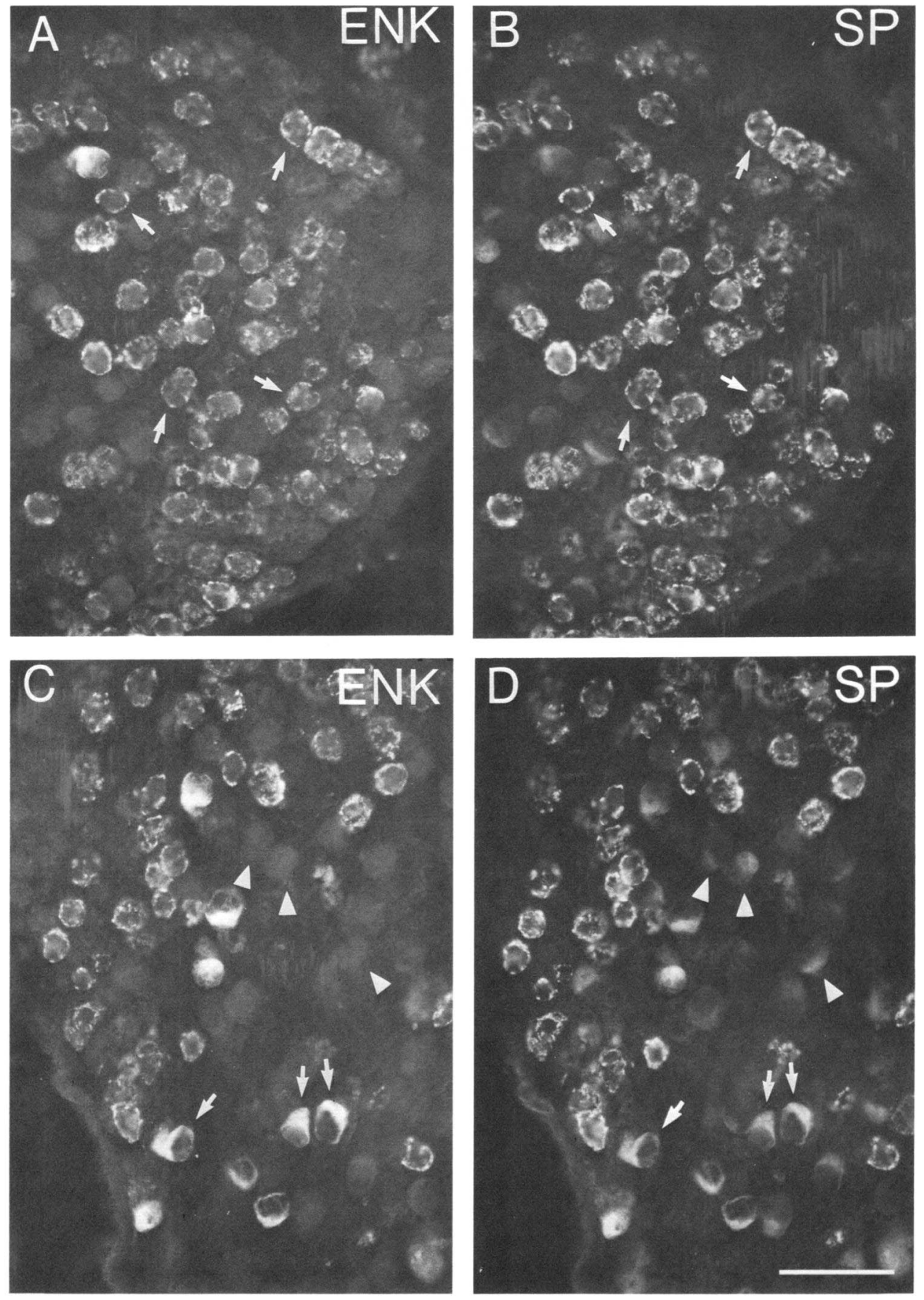

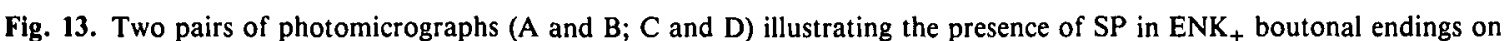
choroidal neurons ( $A$ and $B$ ) and cap-like endings on ciliary neurons ( $C$ and $D$ ), as revealed using immunofluorescence doublelabeling procedures. Photomicrographs A and B show the same field of view from a single section double-labeled for SP and ENK, with the arrows in A indicating some of the choroidal neurons receiving ENK + boutonal endings that are also labeled for SP, as shown in B. Note that nearly all choroidal neurons receive boutonal endings that contain both ENK and SP. Photomicrographs $C$ and D show a single field of view from a section that had been double-labeled for ENK and SP, with the arrows in $C$ indicating some of the ciliary neurons that receive ENK + cap-like endings that also contain SP, as shown in D. Note, however, that not all cap-like endings contain both SP and ENK. Some that contain SP but not ENK are indicated by arrowheads in photomicrographs $C$ and $D$. Scale bar in $D=100 \mu \mathrm{m} ; A-C$ are of the same magnification as in $D$.

counted. Instead, their numbers were assessed by counting the numbers of axons in the postganglionic ciliary and choroidal nerves. Such an approach may not be accurate, however, because (1) individual ciliary ganglion neurons could in principle give rise to more than one axon; and (2) sympathetic and trigeminal fibers appear to enter the eye via the ciliary and choroidal nerves (Marwitt et al., 1971; Cantino \& Mugnaini, 1974; Carpenter \& Sutin, 1983; Reiner, 1987c). We believe our counts 


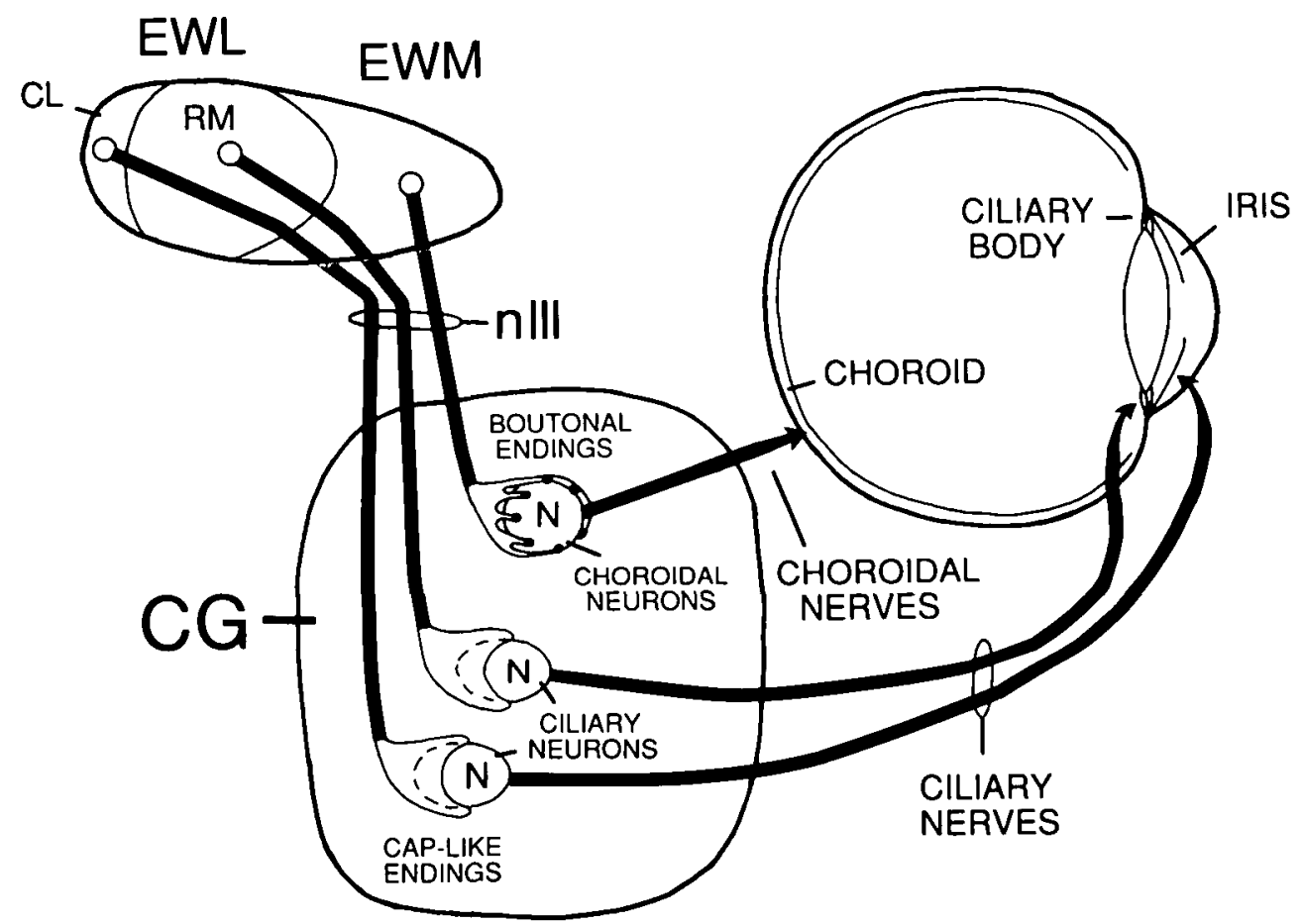

Fig. 14. Summary of the present results. Choroidal neurons, which innervate the choroidal blood vessels and control blood flow, receive boutonal endings from neurons of the ipsilateral EWM. Nearly all of these choroidal neurons receive endings that use SP, ENK, and acetylcholine as neuroactive substances. Ciliary neurons innervating the ciliary body and controlling accommodation are innervated by cap-like endings from neurons of rostromedial (RM) EWL. In contrast, the ciliary neurons innervating the iris musculature and thereby controlling the pupil are innervated by cap-like endings from neurons of caudolateral (CL) EWL. Neurons of EWL appear to use typically either acetylcholine alone, acetylcholine plus SP, or acetylcholine plus SP and ENK as neuroactive substances in influencing ciliary neurons. The majority of neurons of the caudolateral EWL appear to use the latter combination.

to be accurate because we were able to unambiguously identify (because they were ChAT-labeled) and count all ciliary ganglion neurons in a closely spaced series of sections through the ciliary ganglion. Furthermore, we applied Abercrombie's correction to adjust for double counting of cells after measuring the size of the labeled neurons. Moreover, even without the Abercrombie correction, our counts would still be considerably less than the Marwitt et al. (1971) estimate in pigeons. Thus, we believe that the number of neurons in the ciliary ganglion is much less than previously estimated in pigeons. It is interesting to note that our counts for EW and the ciliary ganglion indicate that the number of EWM neurons (approximately 700) is closely matched to the number of their target neurons, the choroidal neurons (approximately 900), as are the EWL neurons (approximately 500) in relation to their target neurons, the ciliary neurons (approximately 600). Since each ciliary neuron receives only a single cap-like ending, these latter results suggest that most individual EWL neurons may innervate only a single ciliary neuron. Although the ratio of EWM neurons to choroidal neurons is also nearly one-to-one, choroidal neurons are known to each receive boutonal endings from two or more EW neurons (Dryer \& Chiappinelli, 1985b).

Finally, in a previous single-label study (Erichsen et al., $1982 a$ ), we estimated the number of ciliary and choroidal neurons of the ciliary ganglion that receive either SP+ or ENK+ preganglionic terminals. To a large extent, those results are in accord with the current results. For example, it was previously reported that $53 \%$ of the ciliary neurons receive SP+ cap-like terminals. The results of the present double-label study indicate that $53.7 \%$ of the ciliary neurons receive SP+ cap-like endings, with about half of these endings also containing ENK. Similarly, it was previously reported that $39 \%$ of the ciliary neurons receive ENK + cap-like endings and in the present study we observed that approximately $30 \%$ of the ciliary neurons receive ENK + cap-like endings, with the vast majority of these also containing SP. In the case of choroidal neurons, the study of Erichsen et al. (1982a) indicated that approximately $50-60 \%$ of the choroidal neurons received SP+ endings and a similar percentage received ENK+ endings. Furthermore, the double-label study of Erichsen et al. (1982 $b$ ) indicated that SP and ENK are typically found in the same endings on choroidal neurons. The present study confirms that SP and ENK co-occur extensively in the boutonal endings, and shows that, in fact, nearly all choroidal neurons (approximately 94\%) receive such SP+/ ENK + boutonal endings. A previous study (Reiner, 1987a) has also reported that the majority of the $\mathrm{SP}+/ \mathrm{ENK}+$ boutonal endings and many of the SP+ or ENK+ cap-like endings also contain vasoactive intestinal polypeptide (VIP).

\section{Functional implications}

\section{Neurotransmitter-distinct populations}

At present, it cannot be certain whether the terminals in the ciliary ganglion that were observed to be nonpeptidergic in the 
present study are a separate population from those that contain one or more peptides. Similarly, it is uncertain if the SP+ terminals and the ENK + terminals are separate populations from the $\mathrm{SP}+/ \mathrm{ENK}+$ terminals. It is possible that all neurons of EW produce SP and ENK, but that the levels of one or more peptide in the EW perikarya and ciliary ganglion terminals are at times below the immunohistochemically detectable level. It is possible that the activity state of EW neurons, in particular, influences the amounts of the different peptides that these neurons synthesize. On the other hand, EW may contain several different types of neurons as defined by neurotransmitter and neuropeptide content and each of these may play a different role in influencing ciliary ganglion neurons. Specific groups of neurons in the ciliary ganglion, particularly ciliary neurons (which each receive input from only one preganglionic ending), may be specialized for responding to input only from a specific population of EW neurons. For example, ciliary neurons receiving input from ENK + cholinergic preganglionic terminals may respond differently to SP than do ciliary neurons receiving $\mathrm{SP}+/$ LENK + cholinergic preganglionic terminals. It will be interesting to know if neurophysiological studies and in situ receptor binding studies also find that either ciliary or choroidal neurons of the ciliary ganglion are subdivided into different subpopulations based on their response properties or receptorbinding characteristics. As yet, little relevant data are available on this point. Since generally consistent percentages of SP-only, LENK-only, and SP/LENK-containing preganglionic terminals were observed in the present study, we favor the view that EW does contain populations of neurons that differ in terms of their neuropeptide content.

\section{Neurophysiology}

Acetylcholine has long been known to be the primary neurotransmitter used by the preganglionic input to the ciliary ganglion, and it is known to depolarize ciliary ganglion neurons of both types (Hess, 1965; Landmesser \& Pilar, 1970, 1978; Pilar \& Tuttle, 1982; Dryer \& Chiappinelli, 1983; Smith et al., 1985; Loring \& Zigmond, 1987; Margiotta et al., 1987; Boyd et al., 1988). A number of recent studies have shown that SP and ENK also affect neurotransmission within the ciliary ganglion. For example, opiate receptors of the mu and delta type have been shown to be present in the ciliary ganglion (Erichsen et al., 1984). Several neurophysiological studies directly support the idea that enkephalin, an endogeneous ligand for these receptors, plays a neuroactive role in the ciliary ganglion (Dryer \& Chiappinelli, 1985a,b,c; Margiotta \& Berg, 1986; Chiappinelli et al., 1989). Enkephalin, however, appears to play a different role in transmission between boutonal endings and choroidal neurons than between cap-like endings and ciliary neurons. Enkephalin (both LENK and MENK) is reported to act postsynaptically in the case of choroidal neurons (Dryer \& Chiappinelli, 1985c), but presynaptically in the case of ciliary neurons (Chiappinelli et al., 1989; Margiotta \& Berg, 1986). In both cases, the effect of enkephalin is to reduce nicotinic transmission through the ganglion. In the case of the choroidal neurons, enkephalin has this effect by directly attenuating the response of choroidal neurons to acetylcholine, while in the case of ciliary neurons, enkephalin may have this effect by a presynaptic action that decreases acetylcholine release. The electrophysiological effects of SP on choroidal and ciliary neurons have also been studied. SP has been consistently found to depolarize choroidal ciliary ganglion neurons (Dryer \& Chiappinelli, 1985a,c). The precise role of SP in the case of cap-like endings on ciliary neurons, however, is unclear since studies of isolated whole ciliary ganglia indicate that SP acts presynaptically to depolarize cap-like endings, but has little if any postsynaptic effect, while studies of cultured dissociated ciliary ganglia cells have found that SP acts directly on ciliary neurons to shorten the after-hyperpolarization to acetylcholine (Dryer \& Chiappinelli, 1985a,b,c; Margiotta \& Berg, 1986; Chiappinelli et al., 1989; Role, 1984). Both of these actions, however, may serve in different ways to maintain the sensitivity of ciliary neurons to acetylcholine. For example, depolarization of the cap-like endings might attenuate the release of acetylcholine from these endings, while shortening the after-hyperpolarization to acetylcholine could decrease the refractory period of these neurons to the depolarizing effects of acetylcholine. Both of these actions of SP and ENK may serve to prevent prolonged hyperpolarization (via activation of calcium-activated potassium channels) of the ciliary neurons due to excessively high preganglionic firing rates. As a result, ciliary neurons might be better able to follow firing of the preganglionic input at high frequencies. These neurons are known to follow activation of their input up to very high frequencies (such as $100 \mathrm{~Hz}$ ), and a number of specializations, such as the presence of electrical coupling, myelin-sheathing of presynaptic and postsynaptic axons, myelin sheathing of ciliary neurons, and single-innervation of ciliary neurons, are thought to play a role in making this possible (Dryer \& Chiappinelli, 1985a,b,d). It is possible that the presence of SP and ENK in cap-like endings is a further adaptation that makes such high-frequency spiking activity possible. Data from ultrastructural anatomical studies are also consistent with the notion that these peptides play their role at high firing frequencies of the preganglionic terminals. Such studies have shown that SP and ENK are contained in large dense core vesicles in these terminals (Erichsen et al., 1982a), and these vesicles are preferentially released from the cap-like endings by high-frequency stimulation $(100 \mathrm{~Hz})$ of the preganglionic input to the ganglion, but not by low-frequency stimulation $(10 \mathrm{~Hz})$ (Philippe \& Tremblay, 1981, 1983; Tremblay \& Philippe, 1981).

\section{Functional significance of co-occurrence}

SP, ENK, and acetylcholine all appear to play a role in neurotransmission through the ciliary ganglion. Previous electrophysiological studies have not, however, examined the possible interactions between SP and ENK in neurotransmission. To some extent, such co-occurrence appears paradoxical. For example, since SP and ENK have opposite influences on choroidal neurons, it is unclear how both could play a role in neurotransmission without canceling the effects of one another. Thus, the functional significance of such co-occurrence in boutonal endings is uncertain. To further resolve this issue, it will be important to determine (1) the stimulation conditions under which SP and ENK are released from boutonal endings; and (2) how SP, ENK, and acetylcholine interact in influencing choroidal neurons. Furthermore, VIP has also been found in numerous neurons of EWM and in boutonal endings on the majority of choroidal neurons (Reiner, 1987a). Consequently, it is also important to elucidate the role of VIP with respect to SP, ENK, and acetylcholine at the synapses between EWM neurons and choroidal neurons to determine the mechanisms of neurotransmission through the ciliary ganglion. Choroidal neurons of the ciliary ganglion control choroidal blood flow and precise regulation of this control may be important for maintaining a 
steady supply of oxygen and nutrients to the retina (Reiner et al., 1990; Fitzgerald et al., 1990a,b). It will be important to determine the role the different neuroactive substances in boutonal endings play in the control of choroidal neurons, and in the control of choroidal blood flow.

Although the functional significance of the co-localization of SP and ENK in preganglionic terminals for the control of ciliary neurons by cap-like endings is not clear, anatomical and physiological data, as discussed above, suggest that these peptides play a role at higher firing frequencies of the preganglionic terminals (Philippe \& Tremblay, 1981,1983; Tremblay \& Philippe, 1981; Erichsen et al., 1982a; Role, 1984; Dryer \& Chiappinelli, 1985a,b,c; Margiotta \& Berg, 1986; Chiappinelli et al., 1989). Whether the postsynaptic response to SP is influenced by ENK and vice versa is, however, currently unknown.

The particular physiological significance of the apparently greater abundance of SP+ cholinergic neurons in the accommodative subdivision of EW and of ENK+ cholinergic neurons in the pupilloconstrictive subdivision of EW is uncertain. Furthermore, the role of the various enkephalin peptides other than LENK in the ciliary ganglion is also uncertain. Finally, the role of VIP at the synapses between the cap-like endings and the ciliary neurons also requires further study, in and of itself and with respect to SP, ENK, and acetylcholine. Nonetheless, the demonstrated presence of these diverse neuropeptides in preganglionic endings and the presence of at least some of their appropriate receptors in the ciliary ganglion clearly indicate that the ciliary ganglion is one site at which a variety of specific pharmacological agents, such as the opiates, may act to influence pupil dilation, accommodation, or the regulation of choroidal blood flow.

\section{Comparison to mammals}

\section{Location of the nucleus of $E W$}

The present studies in pigeons reveal a number of features of the location of EW and the organization of EW that appear to be common among birds, but not necessarily common among mammals. For example, in birds, EW is a cytoarchitectonically distinct cell group located dorsolateral to the somatic subdivisions of the oculomotor nuclear complex. The neurons of this cell group have now been shown to be cholinergic in pigeons and chickens by means of ChAT immunohistochemistry (Sorenson et al., 1989), and this cell group appears cytoarchitectonically identifiable in all birds studied (Ariens-Kappers et al., 1936; Yoshida, 1953; Narayanan \& Narayanan, 1976). In pigeons and chickens (Lyman \& Mugnaini, 1980), the neurons of EW have been shown to be the sole source of the preganglionic input to the ciliary ganglion, and the neurons of EW have not been found to project to any sites within the avian central nervous system (Cabot et al., 1982). These findings in birds contrast strongly with those in mammals. Although a nucleus of Edinger-Westphal has long been recognized in mammals and thought to give rise to the preganglionic input to the ciliary ganglion (reviewed in Reiner et al., 1983a; Evinger, 1988), recent retrograde-labeling studies in felines and rodents have shown that the cell groups identified as EW in these animal groups do not project to the ciliary ganglion but instead project to the spinal cord. Based on retrograde-labeling data, the preganglionic neurons projecting to EW in cats are located along the midline medial and inferior to the somatic subdivisions of the oculomotor nuclear complex (Loewy et al., 1978; Toyoshima et al.,
1980). Retrograde-labeling studies in monkeys indicate that the location and extent of EW in primates may be more similar to that in birds than is true for other studied mammalian species (Akert et al, 1980; Burde \& Loewy, 1980; Reiner et al., 1983a; Clarke et al., 1985). Our own unpublished studies using antiChAT in rhesus monkeys (A. Reiner and P.D.R. Gamlin, unpublished observations) further supports the view that the neurons of EW are cholinergic and have the same general topographic location and cytoarchitectonic distinctness as in birds. Our current studies and our previous findings, as discussed above, also support the view that the preganglionic neurons controlling the pupil, lens, and choroidal blood vessels are located in different parts of the avian EW. In mammals, EW also appears to control the lens, pupil, and choroidal blood vessels (Reiner et al., 1983a; Gherezghiher et al., 1989; Stjernschantz et al., 1976; Stjernschantz \& Bill, 1979). There are currently, however, limited data available for any mammalian species that unequivocally show that such functions in mammals are controlled by different subdivisions in EW. Based on the avian data, it is tempting to speculate that such subdivisions are present in primates, and possibly other mammals as well. The EW stimulation studies of Jampel (1960; Jampel \& Mindel, 1967) in monkeys are consistent with the inference that more rostral parts of EW control accommodation, while the more caudal parts control the pupillary light reflex, as we have found to be true in birds.

\section{Neuropeptides in the preganglionic input to $E W$}

Although both birds and mammals use acetylcholine as the neurotransmitter in the EW input to the neurons of the ciliary ganglion (Gallagher et al., 1982; Hara et al., 1982; Davis et al., 1984), birds (at least pigeons and chickens) and mammals appear to differ in terms of the neuropeptides utilized in this projection. As discussed here and in previous papers, SP, ENK, and VIP are all present in many of the preganglionic inputs from EW in birds. In contrast, this has not been observed in mammals. Nonetheless, neuropeptides do appear to be present in the preganglionic input to the ciliary ganglion and/or have physiological effects on ciliary ganglion neurons in at least some mammals, although the precise peptides present may show some variability from one mammalian species to another (Kondo et al., 1982; Katayama \& Nishi, 1984; Grimes et al., 1990). Thus, the precise neuropeptides present in the EW projection to the ciliary ganglion appears evolutionarily labile. In light of this, it will be interesting to determine if some specific adaptations in the control of lens, pupil, or choroidal blood flow in birds are somewhat uniquely dependent on the neuropeptides localized in this circuit.

\section{Acknowledgments}

We gratefully acknowledge the excellent technical or illustrative assistance of Chris Laverack, Pat Lindaman, Valerie Frye, Joan Speh, Gary Henderson, Debora Romeo, Lynn Cutler, Betty Cook, Donna Malysz, Laura Schramm, and Angela Gero. This research was supported by NS19620 and EY-05298 (A.R.), EY-04587 (J.T.E.), EY-07391 (C.E.), HL24103 (J.B.C.), and EY-01246 (H.J.K.).

\section{References}

Abercrombie, M. (1946). Estimation of nuclear population from microtome sections. Anatomical Record 94, 239-247.

Akert, K., Glickman, M.A., LANG, W., Grob, P. \& Huber, A. (1980). 
The Edinger-Westphal nucleus in the monkey. A retrograde tracer study. Brain Research 184, 491-498.

ANDERSON, K.D. \& ReINER, A. (1990a). The extensive co-occurrence of substance $P$ and dynorphin in striatal projection neurons: an evolutionarily conserved feature of basal ganglia organization. Journal of Comparative Neurology 295, 339-369.

Anderson, K.D. \& Reiner, A. (1990b). The distribution and relative abundance of neurons in the pigeon forebrain containing somatostatin, neuropeptide Y, or both. Journal of Comparative Neurology 299, 261-282.

Ariens-Kappers, C.U., Huber, G.C. \& Crosby, E.C. (1936). The Comparative Anatomy of the Nervous System of Vertebrates, including Man. New York: McMillan Co.

Armstrong, E. (1982). A look at relative brain size in mammals. Neuroscience Letters 34, 101-104.

Bloch, B., Baird, A., Ling, N., Benort, R. \& Guillemin. R. (1983). Immunohistochemical evidence that brain enkephalins arise from a precursor similar to adrenal preproenkephalin. Brain Research 263. $251-257$.

Boyd, R.T., Jacob, M.H., Couturier, S., Ballivet, M. \& Berg, D.K. (1988). Expression and regulation of neuronal acetylcholine receptor mRNA in chick ciliary ganglia. Neuron 1, 495-502.

Brecha, N.C. \& KarTen, H.J. (1981). Organization of the avian accessory optic system. Annals of the New York Academy of Sciences 374, $215-229$.

Burde, R.M. \& LoewY, A.D. (1980). Central origin of oculomotor parasympathetic neurons in the monkey. Brain Research 198, 434-439.

CABot, J.B., Reiner, A. \& Bogan, N. (1982). Avian bulbospinal pathways: anterograde and retrograde studies of cells of origin, funicular trajectories, and laminar terminations. In Progress in Brain Research, ed. KuYPERS, H.G.J.M. \& MARTin, G.F. pp. 79-108. New York: Elsevier.

Cantino, D. \& Mugnaini, E. (1974). Adrenergic innervation of the parasympathetic ciliary ganglion in the chick. Science 185, 279-280.

Carpenter, M.B. \& Sutin, J. (1983). Human Neuroanatomy, 8th edition. Baltimore, Maryland: Williams and Wilkins.

Chiappinelli, V.A., Feng, C. \& MCMahon, L. (1989). Presynaptic responses to opioid peptides and substance $P$ in the avian ciliary ganglion. Investigative Ophthalmology and Visual Science (Suppl) 30, 125.

Clatree, R.J., Colmbra, C.J.P. \& Alessio, M.L. (1985). Distribution of parasympathetic motorneurones in the oculomotor complex innervating the ciliary ganglion in the marmoset (Callithrix jacchus). Acta Anatomica 121, 53-58.

Cowan, W.M. \& WENGER, E. (1968). Degeneration in the nucleus of origin of the preganglionic fibers to the chick ciliary ganglion following early removal of the optic vesicle. Journal of Experimental Zoology 168, 105-124.

Cuello, A.C., Galfre, G. \& Milstein, C. (1979). Detection of substance $\mathrm{P}$ in the central nervous system by a monoclonal antibody. Proceedings of the National Academy of Sciences of the U.S.A. 76, 3532-3536.

Cuello, A.C., Mrstein, C., Coutre, R., Wright, B., Priestleey, J.V. \& JARVIS, J. (1984). Characterization and immunocytochemical application of monoclonal antibodies against enkephalins. Journal of Histochemistry and Cytochemistry 32, 947-957.

Davis, R., Koelle, G.B. \& Sanville, U.J. (1984). Electron-microscopic localization of acetylcholinesterase and butyrylcholinesterase in the ciliary ganglion of the cat. Journal of Histochemistry and Cytochemistry 32, 849-861.

DRYER, S.E. \& ChIAPPINELLI, V.A. (1983). Kappa-bungarotoxin: an intracellular study demonstrating blockade of neuronal nicotinic receptors by a snake neurotoxin. Brain Research 289, 317-321.

Dryer, S.E. \& Chiappinelli, V.A. (1985a). Substance P depolarizes nerve terminals in an autonomic ganglion. Brain Research 336, 190-194.

DRYer, S.E. \& Chiappinelli, V.A. (1985b). Properties of choroid and ciliary neurons in the avian ciliary ganglion and evidence for substance $P$ as a neurotransmitter. Journal of Neuroscience 5, 2654-2661.

DRYer, S.E. \& ChiAPpinelli, V.A. (1985c). Electrophysiological evidence for substance $\mathbf{P}$ as a neurotransmitter in the ciliary ganglion. Society for Neuroscience Abstracts 11, 707.

DRYER, S.E. \& ChIAPPINELli, V.A. (1985d). An intracellular study of synaptic transmission and dendritic morphology in sympathetic neurons of the chick embryo. Developmental Brain Research 22, 99-111.

ERICHSEN, J.T. \& EVINGER, C. (1985). Transsynaptic retrograde studies of the nucleus of Edinger-Westphal and the oculomotor system. Society for Neuroscience Abstracts 11, 1040.

Erichsen, J.T. \& EVINGER, C. (1989). A unique subpopulation of medial rectus motorneurons and its relationship with the nucleus of Edinger-Westphal. Society for Neuroscience Abstracts 15, 240.

ERICHSEN, J.T., KARTEN, H.J., EldRED, W.D. \& BreCha, N.C. (1982a). Localization of substance P-like and enkephalin-like immunoreactivity within preganglionic terminals of the avian ciliary ganglion: light and electron microscopy. Journal of Neuroscience 2, 994-1003.

Erichsen, J.T., Reiner, A. \& Karten, H.J. (1982b). The co-occurrence of substance P-like and leucine-enkephalin-like immunoreactives in neurons and fibers of the avian nervous system. Nature (London) $295,407-410$.

Erichsen, J.T., Keyser, K.T., Zukin, R.S. \& KarTen, H.J. (1984). Opiate receptors: characterization in the avian ciliary ganglion. Society for Neuroscience Abstracts 10, 989.

Evinger, C. (1988). Extraocular motor nuclei: location, morphology, and afferents. In Neuroanatomy of the Oculomotor System, ed. ButtNer-EnNeVer, J.A. pp. 81-117. New York: Elsevier Science Publishers.

Fitzgerald, M.E.C., Vana, B.A. \& Reiner, A. (1990a). Evidence for retinal pathology following interruption of neural regulation of choroidal blood flow: Müller cells express GFAP following lesions of the nucleus of Edinger-Westphal in pigeons. Current Eye Research 9(6), 583-598.

FitzGerald, M.E.C., Vana, B.A. \& Reiner, A. (1990 b). Control of choroidal blood flow by the nucleus of Edinger-Westphal: a laserDoppler study. Investigative Ophthalmology and Visual Science 31, 2483-2492.

Gallagher, J.P., Griffith, W.H. \& Schinnick-Gallagher, P. (1982). Cholinergic transmission in cat parasympathetic ganglia. Journal of Physiology (London) 332, 473-486.

Gamlin, P.D.R., Reiner, A., Erichsen, J.T., Cohen, D.H. \& Karten, J.J. (1984). The neural substrate for the pupillary light reflex in pigeons. Journal of Comparative Neurology 226, 523-543.

Gamlin, P.D.R., Reiner, A., KarTen, H.J. (1982). Substance P-containing neurons of the avian suprachiasmatic nucleus project directly to the nucleus of Edinger-Westphal. Proceedings of the National Academy of Sciences of the U.S.A. 79, 3891-3895.

GherezghiHer, T., Hey, J. \& Koss, M. (1989). Cholinergic control of intraocular pressure. Investigative Ophthalmology and Visual Science (Suppl.) 30, 20.

Grimes, P.A., McGlinN, A. \& Stone, R.A. (1990). Neuropeptide localization in cat ciliary ganglion. Investigative Ophthalmology and Visual Sciences (Suppl.) 31, 40.

Hara, H., Kobayashi, S., Sugita, K. \& Tsukahara, S. (1982). Innervation of dog ciliary ganglion. Histochemistry 76, 295-301.

Hess, A. (1965). Developmental changes in the structure of the synapse on the myelinated cell bodies of the chicken ciliary ganglion. Journal of Cell Biology 25, 1-19.

Itoh, K., Konishi, A., Nomura, S., Mizuno, N., Nakamura, Y. \& Sugimoto, T. (1979). Application of coupled oxidation reaction to electron-microscopic demonstration of horseradish peroxidase: cobalt-glucose oxidase method. Brain Research 175, 341-346.

JAMPEL, R.S. (1960). Convergence, divergence, pupillary reactions, and accommodation of the eyes from Faradic stimulation of the macaque brain. Journal of Comparative Neurology 115, 371-400.

JAMPEL, R.S. \& MiNDEL, J. (1967). The nucleus for accommodation in the midbrain of the macaque. Investigative Ophthalmology 6, 40-50.

Johnson, D.C. \& Epstein, M.L. (1986). Monoclonal antibodies and polyvalent antiserum to chicken choline acetyltransferase. Journal of Neurochemistry 46, 968-976.

KARTEN, H.J. \& Hodos, W. (1967). A Stereotaxic Atlas of the Brain of the Pigeon (Columba livia) Baltimore, Maryland: The Johns Hopkins Press.

KataYAma, Y. \& Nishi, S. (1984). Sites and mechanisms of actions of enkephalin in the feline parasympathetic ganglion. Journal of Physiology (London) 351, 111-121.

Khachaturian, H., Lewis, M.E. \& Watson, S.J. (1983). Co-localization of proenkephalin peptides in rat brain regions. Brain Research 279, 369-373. 
Kondo, H., Katayama, Y. \& YUI, R. (1982). On the occurrence and physiological effect of somatostatin in the ciliary ganglion of cats. Brain Research 247, 141-144.

LANDMEsser, L. \& PilaR, G. (1970). Selective reinnervation of two cell populations in the adult pigeon ciliary ganglion. Journal of Physiology (London) 211, 203-216.

LANDmesser, L. \& Pilar, G. (1974). Synapse formation during embryogenesis on ganglion cells lacking a periphery. Journal of Physiology (London) 241, 715-736.

LANDMEsSER, L. \& Pilar, G. (1978). Interactions between neurons and their targets during in vivo synaptogenesis. Federation Proceedings 37, 2016-2022

LindBerG, I. (1986). On the evolution of proenkaphalin. Trends in Pharmacological Science 7, 216-217.

LOEWY, A.D., SAPER, C.B. \& YAMOdIS, N.D. (1978). Re-evaluation of the efferent projections of the Edinger-Westphal nucleus. Brain Research 141, 153-159.

LORING, R.H. \& ZIGMOND, R.E. (1987). Ultrastructural distribution of [125]-toxin binding sites on chick ciliary neurons: synaptic localization of a toxin that blocks ganglionic nicotinic receptors. Journal of Neuroscience 7, 2153-2162.

LYMAN, D. \& MUGNaINI, E. (1980). The avian accessory oculomotor nucleus. Society for Neuroscience Abstracts 6, 479.

MargiotTA, J.F. \& BerG, D.K. (1986). Enkephalin and substance P modulate synaptic properties of chick ciliary ganglion neurons in cell culture. Neuroscience 18, 175-182.

Margiotta, J.F., Berg, D.K. \& Dionne, V.E. (1987). The properties and regulation of functional acetylcholine receptors on chick ciliary ganglion neurons. Journal of Neuroscience 7, 3612-3622.

MarwitT, R., Pilar, G. \& Weakly, J.N. (1971). Characterization of two ganglion cell populations in avian ciliary ganglion. Brain $R e-$ search 25, 317-334.

McLean, I.W. \& Nakane, P.K. (1974). Periodate-lysine-paraformaldehyde fixative: a new fixative for immunoelectron microscopy. Journal of Histochemistry and Cytochemistry 22, 1077-1083.

Mesulam, M.-M. (1978). Tetramethylbenzidine for horseradish peroxidase neurochemistry: a noncarcinogenic blue reaction product with superior sensitivity for visualizing afferents and efferents. Journal of Histochemistry and Cytochemistry 26, 106-117.

Millar, T.J., Ishimoto, I., Epstein, M.L., Johnson, C.D. \& Morgan, I.G. (1987). Cholinergic amacrine cells of the chicken retina: a lightand electron-microscope immunocytochemical study. Neuroscience 21, 725-743.

Narayanan, C.H. \& Narayanan, Y. (1976). An experimental inquiry into the central source of preganglionic fibers to the chick ciliary ganglion. Journal of Comparative Neurology 166, 101-109.

Philippe, E. \& Tremblay, J.P. (1981). In Vivo stimulation of a cholinergic synapse of the chick ciliary ganglion induces a reduction in the number of dense core vesicles. Neuroscience Letters 24, 307-312.

Philippe, E. \& Tremblay, J.P. (1983). Increased number pre-area of peptidergic and cholinergic vesicles in synapses of the chick ciliary ganglion following $10 \mathrm{~Hz}$ in vivo stimulation. Neuroscience Letters $35,149-154$.

Pilar, G., Landmesser, L. \& Burstein, L. (1980). Competition for survival among developing ciliary ganglion cells. Journal of Neurophysiology 43, 233-254.

PILAR, G. \& TUTTle, J.B. (1982). A simple neuronal system with a range of uses: the avian ciliary ganglion. In Progress in Cholinergic Biology: Model Cholinergic Synapses ed., Goldberg, A. \& Hanin, I. pp. 213-247. New York: Raven Press.

PILAR, G. \& Vaughn, P.C. (1969). Electrophysiological investigations of the pigeon iris neuromuscular junctions. Comparative Biochemistry and Physiology 29, 51-72.

Reiner, A. (1986). The co-occurrence of substance P-like immunoreactivity and dynorphin-like immunoreactivity in striatopallidal and straitonigral projection neurons in birds and reptiles. Brain Research $371,155-161$.

ReINER, A. (1987a). A VIP-like peptide co-occurs with substance P and enkephalin in cholinergic preganglionic terminals of the avian ciliary ganglion. Neuroscience Letters 78, 22-28.

ReINER, A. (1987b). The distribution of proenkephalin-derived peptides in the central nervous system of turtle. Journal of Comparative Neurology 259, 65-91.

REINER, A. (1987c). The presence of substance P/CGRP-containing fibers, VIP-containing fibers and numerous cholinergic fibers on blood vessels of the avian choroid. Investigative Ophthalmology and Visual Science (Suppl.) 28, 81.

ReINER, A. \& CARRAWAY, R.C. (1987). Immunohistochemical and biochemical studies on Lys8-Asn9-Neurotensin8-13 (LANT6)-related peptides in the basal ganglia of pigeons, turtles and hamster. Journal of Comparative Neurology 257, 453-476.

Reiner, A., Davis, B.M., Brecha, N.C. \& Karten, H.J. (1984). The distribution of enkephalin-like immunoreactivity in the telencephalon of the adult and developing domestic chicken. Journal of Comparative Neurology 228, 245-262.

Reiner, A., Eldred, W.D., Beinfeld, M.C. \& Krause, J.E. (1985). The co-occurrence of a substance $P$-like peptide and cholecystokinin-8 in a fiber system of turtle cortex. Journal of Neuroscience 5 , $1522-1526$.

Reiner, A., Fitzgerald, M.E.C. \& Gamlin, P.D.R. (1990). Central neural circuits controlling choroidal blood flow: a laser-Doppler study. Investigative Ophthalmology and Visual Science (Suppl.) 31, 38.

Reiner, A., Karten, H.J., Gamlin, P.D.R. \& Erichsen, J.T. (1983a). Parasympathetic control of ocular function: functional subdivisions and connections of the avian nucleus of Edinger-Westphal. Trends in Neuroscience 6, 140-145.

Reiner, A., Karten, H.J. \& Solina, A.R. (1983b). Substance P: localization within paleostriatal-tegmental pathways in pigeons. $\mathrm{Neu}$ roscience 9, 61-85.

Role, L.W. (1984). Substance of P modulation of acetylcholine-induced currents in embryonic chicken sympathetic and ciliary ganglion neurons. Proceedings of the National Academy of Sciences of the U.S.A. 81, 2924-2928.

Smith, M.A., Stollberg, J., Lindstrom, J.M. \& Berg, D.K. (1985). Characterization of a component in chick ciliary ganglia that crossreacts with monoclonal antibodies to muscle and electric organ acetylcholine receptor. Journal of Neuroscience 5, 2726-2731.

Sorenson, E.M., Parkinson, D., Dahl, J.L \& Chiappinelli, V.A. (1989). Immunohistochemical localization of choline acetyltransferase in the chicken mesencephalon. Journal of Comparative Neurology 281, 641-657.

Stjernschantz, J., Alm, A. \& Bill, A. (1976). Effects of intracranial oculomotor nerve stimulation on ocular blood flow in rabbits: modification by indomethacin. Experimental Eye Research 23, 461-469.

StJERNschantz, J. \& BiLl, A. (1979). Effect of intracranial stimulation of the oculomotor nerve on ocular blood flow in the monkey, cat, and rabbit. Investigative Ophthalmology and Visual Science 18, 99-103.

Terzuolo, C.A. (1951). Richerche sul ganglio ciliare degli Uccelli. Connesssioni e mutamenti in relazione all' eta' e dopo recisione delle fibre pergangliari. Zum Zellforschung und Mikroskopische Anatomie 36, 255-267.

Toyoshima, K., Kawana, E. \& Sakai, H. (1980). On the neuronal origin of the afferents to the ciliary ganglion in the cat. Brain Research 185, 67-76.

Tremblay, J.P. \& Philippe, E. (1981). Morphological changes in presynaptic terminals of the chick ciliary ganglion after stimulation in vivo. A stereological study showing a net loss of total membrane. Experimental Brain Research 43, 439-446.

WESSENDORF, M. \& EldE, R.P. (1985). Characterization of an immunofluorescence technique for the demonstration of co-existing neurotransmitters within nerve fibers and terminals. Journal of Histochemistry and Cytochemistry 33, 984-994.

White, J.D., Krause, J.E., Karten, H.J. \& MCKelvy, J.F. (1985). Presence and ontogeny of enkephalin and substance $P$ in the chick ciliary ganglion. Journal of Neurochemistry 45, 1319-1322.

Wikler, K.C., Perez, G. \& Finlay, B.L. (1989). Duration of retinogenesis: its relationship to retinal organization in two cricetine rodents. Journal of Comparative Neurology 285, 157-176.

Williams, R.G. \& Dockray, G.J. (1982). Differential distribution of Met-enkephalin and Met-enkephalin-Arg6-Phe7-like peptides revealed by immunohistochemistry. Brain Research 240, 167-170.

Williams, R.G. \& Dockray, G.J. (1983). Distribution of enkephalinrelated peptides in rat brain: immunohistochemical studies using antisera to met-enkephalin and met-enkephalin Arg6Phe7. Neuroscience 9, 563-586.

YoshidA, K. (1953). Comparative anatomical and experimental studies on the oculomotor nucleus and neighboring nuclei. Acta Medica et Biologica 1, 143-161. 